\title{
A stabilised Petrov-Galerkin formulation for linear tetrahedral elements in compressible, nearly incompressible and truly incompressible fast dynamics
}

\author{
Antonio J. Gil ${ }^{1}$, Chun Hean Lee, Javier Bonet, Miquel Aguirre \\ Civil and Computational Engineering Centre, College of Engineering \\ Swansea University, Singleton Park, SA2 8PP, United Kingdom
}

\begin{abstract}
A mixed second order stabilised Petrov-Galerkin finite element framework was recently introduced by the authors (C.H.Lee, A.J.Gil and J.Bonet. "Development of a stabilised Petrov-Galerkin formulation for conservation laws in Lagrangian fast solid dynamics", CMAME, 268:40-64, 2014). The new mixed formulation, written as a system of conservation laws for the linear momentum and the deformation gradient, performs extremely well in bending dominated scenarios (even when linear tetrahedral elements are used) yielding equal order of convergence for displacements and stresses. In this paper, this formulation is further enhanced for nearly and truly incompressible deformations with three key novelties. First, a new conservation law for the Jacobian of the deformation is added into the system providing extra flexibility to the scheme. Second, a variationally consistent PetrovGalerkin stabilisation methodology is derived. Third, an adapted fractional step method is presented for both incompressible and nearly incompressible materials in the context of nonlinear elastodynamics. For completeness and ease of understanding, these three improvements are presented both in small and large strain regimes, studying the eigenstructure of the resulting systems. A series of numerical examples are presented in order to demonstrate the robustness of the enhanced methodology with respect to the work previously published by the authors.
\end{abstract}

Keywords: Mixed formulation, Petrov-Galerkin, Fractional step,

\footnotetext{
${ }^{1}$ Corresponding author: a.j.gil@swansea.ac.uk
} 
Incompressible, Locking, Geometric conservation law

\section{Introduction}

Classical displacement-based finite element formulations [1-7] are typically employed in industry when simulating complex engineering problems. For these applications, linear tetrahedral elements tend to be preferred when dealing with complex three dimensional geometries, due to the maturity of the existing unstructured mesh generators. However, this methodology presents a number of well-known shortcomings.

First, reduced order of convergence for derived variables (i.e. second order for displacements but first order for stresses), requiring some form of stress recovery procedure if these are of interest $[8,9]$. Second, the performance of these formulations in bending dominated scenarios can be very poor $[10,11]$ yielding unacceptable results. Third, the presence of numerical instabilities in the form of volumetric locking, shear locking and spurious hydrostatic pressure fluctuations $[12,13]$ when large Poisson's ratios are used. This aspect is particularly relevant in the context of biomedical modelling. Fourth, from the time discretisation point of view, Newmark-type methods [14] have a tendency to introduce high frequency noise, especially in the vicinity of sharp spatial gradients and accuracy is degraded once numerical artificial damping is employed [15-19]. These schemes are thus not desirable for shock dominated problems.

Significant efforts have been undertaken to develop effective linear tetrahedral formulations for nearly incompressible solids. Multi-field Fraeijs de Veubeke-Hu-Washizu (FdVHW) type variational principles [20] are among them, where independent kinematic descriptions are used for the volumetric and deviatoric components of the deformation. The conventional mean dilatation method [21] is a particular case of Selective Reduced Integration, where the volumetric deformation is suitably underintegrated [15]. Unfortunately, the mean dilatation approach cannot be employed with linear tetrahedrals and authors resort to some form of projection to reduce the number of volumetric constraints [21-29].

A family of nodally integrated tetrahedral elements was formulated in [30], where the volumetric strain energy functional was approximated through averaged nodal pressures. However, the resulting approach was reported to behave poorly in bending dominated scenarios. To overcome this difficulty, 
reference [11] proposed the nodal based uniform strain tetrahedral element by applying a nodal averaging process to the whole small strain tensor. Reference [31] extended this application to the large strain regime with the idea of employing both a nodal average Jacobian and a nodal average deformation gradient in the calculation of the stress tensor. As reported in [32-35], the resulting formulation suffers from artificial mechanisms similar to hourglassing unless some form of stabilisation is used. Despite exhibiting very good behaviour in terms of displacements, this class of averaged nodal strain tetrahedral formulations tend to exhibit non-physical hydrostatic pressure fluctuations $[32,36]$.

In parallel, in Reference [37], a stabilised Petrov-Galerkin (PG) formulation by using the Galerkin Least Squares (GLS) approach is first introduced for the analysis of the Stokes problem, with equal order of interpolation for velocity and pressure. The formulation circumvents the LadyzenskayaBabuska-Brezzi (LBB) condition [38, 39], ensuring numerical stability and optimal convergence.

At present and to the best of our knowledge, most of the proposed schemes for linear tetrahedral elements are restricted to elastostatics [32, 40-43]. The development of an effective linear tetrahedral formulation in the range of fully and nearly incompressible large strain dynamics remains an open issue.

The aim of this paper is to improve the robustness and effectiveness of the stabilised Petrov-Galerkin (PG) mixed finite element framework recently presented in [44], extending its applicability to the range of fully and nearly incompressible materials. This mixed methodology is formulated in the form of a system of first order conservation laws [10, 44, 45], where the linear momentum $\boldsymbol{p}$ and the deformation gradient tensor $\boldsymbol{F}$ of the system are regarded as the main conservation variables of this mixed $\boldsymbol{p}$ - $\boldsymbol{F}$ approach. In [44], a robust and stable PG implementation is presented, derived with the help of the Variational Multi-Scale (VMS) method [46-49]. Unfortunately, in the case of extreme deformations in the incompressible limit (i.e. refer to twisting column example in Section 4.4 of this paper), the $\boldsymbol{p}$ - $\boldsymbol{F}$ formulation lacks robustness.

With this in mind, this mixed PG formulation is first enhanced by introducing a new conservation law for the Jacobian $J$ of the deformation (volumetric strain in the small strain regime), also known as a Geometric Conservation Law [50]. The volumetric stress component appearing in the conservation of linear momentum equation is then evaluated from this new conservation law, providing more flexibility and robustness to the scheme. 
For computational efficiency, the enhanced $\boldsymbol{p}-\boldsymbol{F}-J$ formulation is implemented in conjunction with an explicit time integrator, where the time step size is controlled through the Courant-Friedrichs-Lewy number [51] by the volumetric wave speed $c_{p}$. In the incompressibility limit, $c_{p}$ can reach very high values leading to a very inefficient algorithm. Moreover, in the case of full incompressibility, the explicit $\boldsymbol{p}-\boldsymbol{F}-J$ formulation cannot be used.

In order to address this issue, we then present an alternative adapted variationally consistent fractional step [52-54] PG formulation. This fractional step approach is very typical in the context of Computational Fluid Dynamics for incompressible flows $[55,56]$ and has been already used in the context of large deformation solid dynamics [57]. In this case, the allowable time step is found to depend only on the shear wave speed $c_{s}$, circumventing the volumetric wave speed constraint.

The outline of the present paper is as follows. In Section 2 we start by introducing the enhanced stabilised PG formulation for linear elastodynamics. Governing equations, eigenstructure of the problem and the PG stabilisation is presented. For incompressible or nearly incompressible materials, an alternative fractional step approach is also presented. We then extend these formulations to nonlinear large strain elastodynamics (see Section 3). The governing conservation laws are particularised for the case of a nearly incompressible Neo-Hookean material and the eigenstructure of the problem is studied in detail in order to demonstrate the rank-one convexity requirement [58]. This section ends with the PG stabilisation procedure as well as the alternative fractional step approach. In Section 4, a series of numerical examples are presented to assess the robustness of the enhanced formulations and to draw some comparisons against previous results published by the authors [44]. Finally, Section 5 presents some concluding remarks and current directions of research.

\section{Linear reversible elastodynamics}

\subsection{Enhanced $\boldsymbol{p}-\boldsymbol{G}-j$ mixed methodology}

In the context of small deformations, let us consider the motion of a continuum defined by a domain $v \subset \mathbb{R}^{3}$ of boundary $\partial v$ with outward unit normal $\boldsymbol{n}$. This motion is defined by a displacement field $\boldsymbol{u}=\boldsymbol{u}(\boldsymbol{x}, t)$ where $\boldsymbol{x}$ represents a material point and $t$ the time. Let us also introduce the following scalar, vector and second order tensor variables: $\rho$ is the density of the continuum, $\boldsymbol{p}$ is the linear momentum, $\boldsymbol{G}$ is the displacement gradient 
tensor, $j$ is the volumetric strain, $\boldsymbol{\sigma}$ is the (symmetric) Cauchy stress tensor, $\boldsymbol{b}$ is a body force per unit of mass and $E_{T}$ denotes the total energy per unit of volume. It is then possible to describe the motion of the continuum by means of a system of first order conservation laws as follows

$$
\begin{aligned}
\frac{\partial \boldsymbol{p}}{\partial t}-\operatorname{div} \boldsymbol{\sigma} & =\rho \boldsymbol{b} \\
\frac{\partial \boldsymbol{G}}{\partial t}-\operatorname{div}\left(\frac{1}{\rho} \boldsymbol{p} \otimes \boldsymbol{I}\right) & =\mathbf{0} \\
\frac{\partial j}{\partial t}-\operatorname{div}\left(\frac{\boldsymbol{p}}{\rho}\right) & =0 \\
\frac{\partial E_{T}}{\partial t}-\operatorname{div}\left(\frac{1}{\rho} \boldsymbol{\sigma}^{T} \boldsymbol{p}-\boldsymbol{Q}\right) & =s
\end{aligned}
$$

In above system (1a-1d), div is the divergence operator defined by the tensor contraction of the last index and $\boldsymbol{I}$ is the identity tensor with Kronecker delta components $[\boldsymbol{I}]_{i j}=\delta_{i j}$. Equation (1a) represents the conservation of linear momentum, (1b) and (1c) represent evolution equations for the displacement gradient tensor and the volumetric strain, respectively, and equation (1d) denotes the conservation of the total energy per unit of volume.

In the case of an adiabatic isothermal deformation, the heat flux $\boldsymbol{Q}$ and the heat source $s$ are neglected and equation (1d) is fully decoupled from the rest of the system. Form the computational point of view, this equation is still very useful when evaluating the numerical diffusion (entropy) introduced by the algorithm. The Cauchy stress tensor $\boldsymbol{\sigma}$ is considered a function of $\boldsymbol{G}$ and $j$, which are evaluated through equations (1b) and (1c), respectively. In other words, in this mixed formulation, $\boldsymbol{G}$ and $j$ are not explicitly evaluated through the displacement field, namely $\nabla \boldsymbol{u}$ and $\operatorname{div} \boldsymbol{u}$, respectively ${ }^{2}$. This enhanced $\boldsymbol{p}-\boldsymbol{G}-\boldsymbol{j}$ mixed methodology will render great benefits when dealing with bending dominated nearly or truly incompressible deformations.

Finally, the above system (1a-1c) of conservation laws can be written in a more compact form as

$$
\frac{\partial \mathcal{U}}{\partial t}+\frac{\partial \mathcal{F}_{i}}{\partial x_{i}}=\mathcal{S}, \quad i=1,2,3
$$

where $\mathcal{U}$ represents the vector of conservation variables, $\mathcal{F}_{i}$ is the flux vector

\footnotetext{
${ }^{2} \nabla$ is the gradient operator.
} 
in the spatial direction $i$ and $\mathcal{S}$ is a source term ${ }^{3}$. The components of $\mathcal{U}, \mathcal{S}$ and $\mathcal{F}_{i}$ can be vectorised leading to $13 \times 1$ column vectors as

$$
\mathcal{U}=\left[\begin{array}{c}
p_{1} \\
p_{2} \\
p_{3} \\
G_{11} \\
G_{12} \\
G_{13} \\
G_{21} \\
G_{22} \\
G_{23} \\
G_{31} \\
G_{32} \\
G_{33} \\
j
\end{array}\right], \quad \mathcal{F}_{i}=\left[\begin{array}{c}
-\sigma_{1 i} \\
-\sigma_{2 i} \\
-\sigma_{3 i} \\
-\delta_{i 1} v_{1} \\
-\delta_{i 2} v_{1} \\
-\delta_{i 3} v_{1} \\
-\delta_{i 1} v_{2} \\
-\delta_{i 2} v_{2} \\
-\delta_{i 3} v_{2} \\
-\delta_{i 1} v_{3} \\
-\delta_{i 2} v_{3} \\
-\delta_{i 3} v_{3} \\
-v_{i}
\end{array}\right], \quad \mathcal{S}=\left[\begin{array}{c}
\rho b_{1} \\
\rho b_{2} \\
\rho b_{3} \\
0 \\
0 \\
0 \\
0 \\
0 \\
0 \\
0 \\
0 \\
0 \\
0
\end{array}\right] .
$$

For clarity, individual conservations laws will be used for each component of the vector of conservation variables $\mathcal{U}$ as

$$
\mathcal{U}=\left[\begin{array}{c}
\boldsymbol{p} \\
\boldsymbol{G} \\
j
\end{array}\right], \quad \mathcal{F}_{\boldsymbol{n}}=\mathcal{F}_{i} n_{i}=\left[\begin{array}{c}
-\boldsymbol{\sigma} \boldsymbol{n} \\
\frac{-1}{\rho} \boldsymbol{p} \otimes \boldsymbol{n} \\
\frac{-1}{\rho} \boldsymbol{p} \cdot \boldsymbol{n}
\end{array}\right], \quad \mathcal{S}=\left[\begin{array}{c}
\rho \boldsymbol{b} \\
\mathbf{0} \\
0
\end{array}\right]
$$

For the closure of the above system (1a-1c), a constitutive law is required relating $\boldsymbol{\sigma}$ and $\boldsymbol{G}$ and $j$. In this case, we employ a linear elastic isotropic constitutive law of the form

$$
\boldsymbol{\sigma}=\underbrace{\mu\left(\boldsymbol{G}+\boldsymbol{G}^{T}-\frac{2}{3}(\operatorname{tr} \boldsymbol{G}) \boldsymbol{I}\right)}_{\boldsymbol{\sigma}^{\text {dev }}}+\underbrace{p \boldsymbol{I}}_{\boldsymbol{\sigma}^{\text {vol }}} ; \quad p=\kappa j,
$$

where $\boldsymbol{\sigma}^{d e v}$ and $\boldsymbol{\sigma}^{v o l}$ represent the deviatoric and volumetric components of the Cauchy stress, $p$ is the hydrostatic pressure and $\mu$ and $\kappa$ are the shear and bulk moduli, respectively. Above system (2) can be re-written in quasi-linear format

$$
\frac{\partial \mathcal{U}}{\partial t}+\mathcal{A}_{i} \frac{\partial \mathcal{U}}{\partial x_{i}}=\mathcal{S}, \quad i=1,2,3,
$$

\footnotetext{
${ }^{3}$ Einstein's summation will be implied for repeated indices unless otherwise explicitly stated.
} 
where the corresponding flux Jacobian matrix $\mathcal{A}_{\boldsymbol{n}}=\mathcal{A}_{i} n_{i}$ is evaluated as

$$
\mathcal{A}_{\boldsymbol{n}}=\left[\begin{array}{ccc}
-\frac{\partial(\boldsymbol{\sigma} \boldsymbol{n})}{\partial \boldsymbol{p}} & -\frac{\partial(\boldsymbol{\sigma} \boldsymbol{n})}{\partial \boldsymbol{G}} & -\frac{\partial(\boldsymbol{\sigma} \boldsymbol{n})}{\partial j} \\
-\frac{\partial\left(\frac{1}{\rho} \boldsymbol{p} \otimes \boldsymbol{n}\right)}{\partial \boldsymbol{p}} & -\frac{\partial\left(\frac{1}{\rho} \boldsymbol{p} \otimes \boldsymbol{n}\right)}{\partial \boldsymbol{G}} & -\frac{\partial\left(\frac{1}{\rho} \boldsymbol{p} \otimes \boldsymbol{n}\right)}{\partial j} \\
-\frac{\partial\left(\frac{1}{\rho} \boldsymbol{p} \cdot \boldsymbol{n}\right)}{\partial \boldsymbol{p}} & -\frac{\partial\left(\frac{1}{\rho} \boldsymbol{p} \cdot \boldsymbol{n}\right)}{\partial \boldsymbol{G}} & -\frac{\partial\left(\frac{1}{\rho} \boldsymbol{p} \cdot \boldsymbol{n}\right)}{\partial j}
\end{array}\right]=\left[\begin{array}{ccc}
\mathbf{0}_{3 \times 3} & -\boldsymbol{c}_{\boldsymbol{n}}^{d e v} & -\kappa \boldsymbol{n} \\
-\frac{1}{\rho} \boldsymbol{I}_{\boldsymbol{n}} & \mathbf{0}_{3 \times 3 \times 3 \times 3} & \mathbf{0}_{3 \times 3 \times 1} \\
-\frac{1}{\rho} \boldsymbol{n} & \mathbf{0}_{3 \times 3} & 0
\end{array}\right],
$$

where

$$
\begin{aligned}
{\left[\boldsymbol{c}_{\boldsymbol{n}}^{d e v}\right]_{i j k} } & =\left[\boldsymbol{c}^{d e v}\right]_{i l j k} n_{l}=\frac{\partial\left[\boldsymbol{\sigma}^{d e v}\right]_{i l}}{\partial[\boldsymbol{G}]_{j k}} n_{l}=\mu\left(\delta_{i j} n_{k}+\delta_{i k} n_{j}-\frac{2}{3} \delta_{j k} n_{i}\right), \\
{\left[\boldsymbol{I}_{\boldsymbol{n}}\right]_{i j k} } & =\delta_{i k} n_{j}
\end{aligned}
$$

For completeness, the residuals $\mathcal{R}=\left[\boldsymbol{\mathcal { R }}_{\boldsymbol{p}}, \boldsymbol{\mathcal { R }}_{\boldsymbol{G}}, \mathcal{R}_{j}\right]^{T}$ of the balance principles (1a-1c) can be defined as

$$
\begin{aligned}
\mathcal{R}_{\boldsymbol{p}} & :=\operatorname{div} \boldsymbol{\sigma}+\rho \boldsymbol{b}-\dot{\boldsymbol{p}} \\
\mathcal{R}_{\boldsymbol{G}} & :=\nabla\left(\frac{\boldsymbol{p}}{\rho}\right)-\dot{\boldsymbol{G}} \\
\mathcal{R}_{j} & :=\operatorname{div}\left(\frac{\boldsymbol{p}}{\rho}\right)-\dot{j}
\end{aligned}
$$

where $(\cdot)$ indicates derivative with respect to time. These residuals will be used later in the paper when deriving a stabilised formulation.

\subsection{Eigenvalue structure}

The study of the eigenvalue structure of the system is important to guarantee its hyperbolicity. The eigenvalues and eigenvectors of system (6) can be evaluated considering plane wave solutions of the form

$$
\mathcal{U}=\phi\left(\boldsymbol{x} \cdot \boldsymbol{n}-c_{\alpha} t\right) \overline{\boldsymbol{U}}_{\alpha}=\phi\left(\boldsymbol{x} \cdot \boldsymbol{n}-c_{\alpha} t\right)\left[\begin{array}{c}
\boldsymbol{p}_{\alpha} \\
\boldsymbol{G}_{\alpha} \\
j_{\alpha}
\end{array}\right]
$$

where $c_{\alpha}$ are the eigenvalues (wave speeds) corresponding to the eigenvector $\overline{\mathcal{U}}_{\alpha}$. Substitution of expression (10) into system (6) renders

$$
c_{\alpha} \overline{\mathcal{U}}_{\alpha}=\mathcal{A}_{n} \overline{\mathcal{U}}_{\alpha}
$$


The above system (11) can be expanded as

$$
\begin{aligned}
c_{\alpha} \boldsymbol{p}_{\alpha} & =-\boldsymbol{c}_{\boldsymbol{n}}^{\text {dev }} \boldsymbol{G}_{\alpha}-\kappa j_{\alpha} \boldsymbol{n} \\
c_{\alpha} \boldsymbol{G}_{\alpha} & =\frac{-1}{\rho} \boldsymbol{p}_{\alpha} \otimes \boldsymbol{n} \\
c_{\alpha} j_{\alpha} & =\frac{-1}{\rho} \boldsymbol{p}_{\alpha} \cdot \boldsymbol{n}
\end{aligned}
$$

Substitution of equations (12b) and (12c) into (12a) and after making use of (8a), it renders

$$
\rho c_{\alpha}^{2} \boldsymbol{p}_{\alpha}=\left[\left(\frac{4}{3} \mu+\kappa\right) \boldsymbol{n} \otimes \boldsymbol{n}+\mu(\boldsymbol{I}-\boldsymbol{n} \otimes \boldsymbol{n})\right] \boldsymbol{p}_{\alpha},
$$

where it can be seen that the system contains three pairs of non-zero eigenvalues corresponding to the volumetric and shear waves as

$$
c_{1,2}= \pm c_{p}, c_{p}=\sqrt{\frac{\frac{4}{3} \mu+\kappa}{\rho}}, \quad c_{3,4}=c_{5,6}= \pm c_{s}, c_{s}=\sqrt{\frac{\mu}{\rho}},
$$

with eigenvectors

$\overline{\mathcal{U}}_{1,2}=\left[\begin{array}{c}\boldsymbol{n} \\ \pm \frac{1}{\rho c_{p}} \boldsymbol{n} \otimes \boldsymbol{n} \\ \pm \frac{1}{\rho c_{p}}\end{array}\right], \quad \overline{\mathcal{U}}_{3,4}=\left[\begin{array}{c}\boldsymbol{t}_{1} \\ \pm \frac{1}{\rho c_{s}} \boldsymbol{t}_{1} \otimes \boldsymbol{n} \\ 0\end{array}\right], \quad \overline{\mathcal{U}}_{5,6}=\left[\begin{array}{c}\boldsymbol{t}_{2} \\ \pm \frac{1}{\rho c_{s}} \boldsymbol{t}_{2} \otimes \boldsymbol{n} \\ 0\end{array}\right]$

where $\boldsymbol{t}_{1}$ and $\boldsymbol{t}_{2}$ are two arbitrary tangential vectors orthogonal to $\boldsymbol{n}$. The rest of the eigenvalues are zero and have null associated velocity components. As can be observed, both volumetric and shear waves always take real values, ensuring the hyperbolicity of the system.

For a fully incompressible material, the conservation equation for the volumetric strain (1c) is replaced by the constraint $\operatorname{div}\left(\frac{\boldsymbol{p}}{\rho}\right)=0$ and thus, equation (12c) gets replaced by the constraint $\boldsymbol{p}_{\alpha} \cdot \boldsymbol{n}=0$. This constraint, once substituted into equation (13) yields

$$
\rho c_{\alpha}^{2} \boldsymbol{p}_{\alpha}=\mu \boldsymbol{p}_{\alpha}
$$

leading to only two pairs of non-zero eigenvalues corresponding to the shear waves $c_{3,4}=c_{5,6}= \pm c_{s}$ with eigenvectors $\overline{\mathcal{U}}_{3,4}$ and $\overline{\mathcal{U}}_{5,6}$. 


\subsection{Linearised Petrov-Galerkin formulation}

A stabilised Petrov-Galerkin (PG) framework incorporating suitable numerical diffusion into the standard Bubnov-Galerkin formulation is presented next. First, we derive a single variational statement that satisfies the second law of thermodynamics $[59,60]$ through the use of work-conjugate principles [61]. Multiplication of the residuals $\mathcal{R}=\left[\boldsymbol{\mathcal { R }}_{\boldsymbol{p}}, \boldsymbol{\mathcal { R }}_{\boldsymbol{G}}, \mathcal{R}_{j}\right]^{T}$ with a set of appropriate conjugate virtual fields $\delta \mathcal{V}^{s t}=\left[\delta \boldsymbol{v}^{s t}, \delta \boldsymbol{\sigma}^{s t}, \delta q^{s t}\right]^{T}$ and integration over the domain $v$ gives

$$
\delta W^{\mathrm{PG}}\left(\mathcal{U}, \delta \mathcal{V}^{s t}\right)=\int_{v} \delta \boldsymbol{v}^{s t} \cdot \mathcal{R}_{p} d v+\int_{v} \delta \boldsymbol{\sigma}^{s t}: \mathcal{R}_{G} d v+\int_{v} \delta q^{s t} \mathcal{R}_{j} d v=0
$$

Note that $\delta \boldsymbol{v}^{s t}$ is the stabilised virtual velocity, $\delta \boldsymbol{\sigma}^{s t}$ is the stabilised virtual Cauchy stress and $\delta q^{s t}$ is the stabilised virtual pressure. Pairs such as $\left\{\delta \boldsymbol{v}^{s t}, \boldsymbol{\mathcal { R }}_{\boldsymbol{p}}\right\},\left\{\delta \boldsymbol{\sigma}^{s t}, \boldsymbol{\mathcal { R }}_{G}\right\}$ and $\left\{\delta q^{s t}, \mathcal{R}_{j}\right\}$ are said to be dual or work conjugate with respect to the volume $v$ in the sense that their inner product yields work rate per unit of volume.

Following the Streamline Upwind Petrov-Galerkin (SUPG) [62-66] approach, stabilised virtual fields can be defined as

$$
\delta \mathcal{V}^{s t}=\delta \mathcal{V}+\boldsymbol{\tau}^{T} \mathcal{A}_{i}^{T} \frac{\partial \delta \mathcal{V}}{\partial x_{i}}
$$

where $\boldsymbol{\tau}$ is the matrix of stabilisation parameters. We can now particularise the above expression (18) to the set of conjugate virtual fields of interest in this paper as

$$
\begin{aligned}
\delta \boldsymbol{v}^{s t} & =\delta \boldsymbol{v}-\frac{\tau_{\boldsymbol{p} \boldsymbol{G}}}{\rho} \operatorname{div} \delta \boldsymbol{\sigma}-\frac{\tau_{\boldsymbol{p} j}}{\rho} \nabla \delta q, \\
\delta \boldsymbol{\sigma}^{s t} & =\delta \boldsymbol{\sigma}-\tau_{\boldsymbol{G p}} \boldsymbol{c}^{d e v}: \boldsymbol{\nabla} \delta \boldsymbol{v}, \\
\delta q^{s t} & =\delta q-\tau_{j \boldsymbol{p}} \kappa \operatorname{div} \delta \boldsymbol{v},
\end{aligned}
$$

where $\boldsymbol{c}^{d e v}$ is the tangent deviatoric constitutive tensor (8a) and $\tau_{p G}, \tau_{\boldsymbol{p} j}, \tau_{\boldsymbol{G} p}$ and $\tau_{j p}$ are appropriate stabilisation parameters. Substitution of equations 
(19a-19c) into (17) yields

$$
\begin{aligned}
& \delta W_{\delta \boldsymbol{v}}^{\mathrm{PG}}(\mathcal{U}, \delta \boldsymbol{v})=\int_{v}\left(\delta \boldsymbol{v} \cdot \mathcal{R}_{\boldsymbol{p}}-\tau_{\boldsymbol{G} \boldsymbol{p}} \boldsymbol{\nabla} \delta \boldsymbol{v}: \boldsymbol{c}^{\text {dev }}: \boldsymbol{\mathcal { R }}_{\boldsymbol{G}}-\tau_{j \boldsymbol{p}} \kappa(\operatorname{div} \delta \boldsymbol{v}) \mathcal{R}_{j}\right) d v=0 \\
& \delta W_{\delta \boldsymbol{\sigma}}^{\mathrm{PG}}(\mathcal{U}, \delta \boldsymbol{\sigma})=\int_{v}\left(\delta \boldsymbol{\sigma}: \boldsymbol{\mathcal { R }}_{\boldsymbol{G}}-\frac{\tau_{\boldsymbol{p} \boldsymbol{G}}}{\rho}(\operatorname{div} \delta \boldsymbol{\sigma}) \cdot \boldsymbol{\mathcal { R }}_{\boldsymbol{p}}\right) d v=0 \\
& \delta W_{\delta q}^{\mathrm{PG}}(\boldsymbol{U}, \delta q)=\int_{v}\left(\delta q \mathcal{R}_{j}-\frac{\tau_{\boldsymbol{p} j}}{\rho}(\boldsymbol{\nabla} \delta q) \cdot \boldsymbol{\mathcal { R }}_{\boldsymbol{p}}\right) d v=0 .
\end{aligned}
$$

Following a standard finite element isoparametric methodology [61], the conservation variables $\mathcal{U}$ as well the virtual fields $\delta \mathcal{V}$ can be discretised in terms of nodal values $\left(\mathcal{U}_{a}, \delta \mathcal{V}_{a}\right)$ and suitable shape functions $N_{a}$, where $a=\{1, \ldots, n\}, n$ being the total number of nodes of the underlying mesh. In our case, linear shape functions are preferred guaranteeing thus second order convergence. It is easier to first consider the discretised weak statement of the linear momentum balance principle $\delta W_{\delta \boldsymbol{v}}^{\mathrm{PG}}(20 \mathrm{a})$. Given the interpolation of the virtual velocity $\delta \boldsymbol{v}=\sum_{a} N_{a} \delta \boldsymbol{v}_{a}$ and the arbitrariness of $\delta \boldsymbol{v}_{a}$, it yields

$$
\begin{aligned}
\int_{v} N_{a} \rho \dot{\boldsymbol{v}} d v & =\int_{\partial v} N_{a} \boldsymbol{t}^{B} d a+\int_{v} N_{a} \rho \boldsymbol{b} d v \\
& -\int_{v} \underbrace{\left[\boldsymbol{\sigma}^{d e v}+\tau_{\boldsymbol{G} p} \boldsymbol{c}^{d e v}: \boldsymbol{\mathcal { R }}_{\boldsymbol{G}}\right]}_{\boldsymbol{\sigma}^{d e v}, s t} \nabla N_{a} d v \\
& -\int_{v} \underbrace{\left[p+\kappa \tau_{j \boldsymbol{p}} \mathcal{R}_{j}\right]}_{p^{s t}} \nabla N_{a} d v,
\end{aligned}
$$

where $\boldsymbol{t}^{B}$ is the boundary traction vector defined as $\boldsymbol{\sigma} \boldsymbol{n}$ (obtained from appropriate boundary conditions). Observe that the squared bracket terms on the right-hand side of (21) describe the stabilised deviatoric Cauchy stress $\boldsymbol{\sigma}^{\text {dev,st }}$ and the stabilised pressure $p^{s t}$. More generally, these stabilised kinetic fields $\boldsymbol{\sigma}^{\text {dev,st }}$ and $p^{\text {st }}$ can be reinterpreted by using the Variational Multi-Scale (VMS) method [46-49, 67-70] as

$$
\boldsymbol{\sigma}^{d e v, s t}:=\boldsymbol{\sigma}^{d e v}\left(\boldsymbol{G}^{s t}\right), \quad p^{s t}:=p\left(j^{s t}\right),
$$

where the stabilised displacement gradient $\boldsymbol{G}^{\text {st }}$ and volumetric strain $j^{\text {st }}$ are 
defined as

$$
\begin{aligned}
\boldsymbol{G}^{s t} & :=\boldsymbol{G}+\tau_{\boldsymbol{G p}} \boldsymbol{\mathcal { R }}_{\boldsymbol{G}}, \\
j^{s t} & :=j+\tau_{j p} \mathcal{R}_{j} .
\end{aligned}
$$

Substitution of equations (22) and (23a-23b) into (21) and after expanding $\dot{\boldsymbol{p}}=\sum_{b} N_{b} \dot{\boldsymbol{p}}_{b}$, results in

$$
\sum_{b} \mathcal{M}_{a b} \dot{\boldsymbol{p}}_{b}=\int_{\partial v} N_{a} \boldsymbol{t}^{B} d a+\int_{v} N_{a} \rho \boldsymbol{b} d v-\int_{v} \boldsymbol{\sigma}\left(\boldsymbol{G}^{s t}, j^{s t}\right) \boldsymbol{\nabla} N_{a} d v,
$$

where $\mathcal{M}_{a b}=\mathcal{M}_{a b} \boldsymbol{I}$ with $\mathcal{M}_{a b}=\left(\int_{v} N_{a} N_{b} d v\right)$ the consistent mass contribution. An identical spatial discretisation procedure can now be followed for $\delta W_{\delta \sigma}^{\mathrm{PG}}(20 \mathrm{~b})$ and $\delta W_{\delta q}^{\mathrm{PG}}(20 \mathrm{c})$ by employing similar finite element expansions $\left\{\delta \boldsymbol{\sigma}=\sum_{a} N_{a} \delta \boldsymbol{\sigma}_{a}, \delta q=\sum_{a} N_{a} \delta q_{a}\right\}$ and $\left\{\dot{\boldsymbol{G}}=\sum_{b} N_{b} \dot{\boldsymbol{G}}_{b}, \dot{j}=\sum_{b} N_{b} \dot{j}_{b}\right\}$, resulting in

$$
\begin{aligned}
\sum_{b} \mathcal{M}_{a b} \dot{\boldsymbol{G}}_{b} & =\int_{\partial v} N_{a}\left(\frac{\boldsymbol{p}^{B}}{\rho} \otimes \boldsymbol{n}\right) d a-\int_{v} \frac{\boldsymbol{p}_{G}^{s t}}{\rho} \otimes \nabla N_{a} d v \\
\sum_{b} \mathcal{M}_{a b} \dot{j}_{b} & =\int_{\partial v} N_{a}\left(\frac{\boldsymbol{p}^{B}}{\rho} \cdot \boldsymbol{n}\right) d a-\int_{v} \frac{\boldsymbol{p}_{j}^{s t}}{\rho} \cdot \nabla N_{a} d v
\end{aligned}
$$

where the stabilised linear momenta $\boldsymbol{p}_{\boldsymbol{G}}^{\text {st }}$ and $\boldsymbol{p}_{j}^{s t}$ are defined by

$$
\begin{aligned}
\boldsymbol{p}_{G}^{s t} & =\boldsymbol{p}+\tau_{p G} \mathcal{R}_{\boldsymbol{p}}, \\
\boldsymbol{p}_{j}^{s t} & =\boldsymbol{p}+\tau_{\boldsymbol{p} j} \boldsymbol{\mathcal { R }}_{\boldsymbol{p}} .
\end{aligned}
$$

In order to speed up the algorithm, the consistent mass matrix contributions are replaced by lumped mass matrix contributions without affecting the order of convergence [44]. In addition, traction and linear momentum vectors at the boundary, denoted as $\boldsymbol{t}^{B}$ (see equation (24)) and $\boldsymbol{p}^{B}$ (see equations (25a-25b)), are computed from prescribed (essential and natural) boundary conditions.

Notice that in applying the stabilisation described in (26a), the displacement gradient will no longer be a discrete spatial gradient (in some weighted residual sense (25a)). Hence, as already presented in [44, 71], it is preferred to adopt $\tau_{p G}=0$, where no stabilisation is added to this term in order to ensure the satisfaction of this involution, namely, $\boldsymbol{G}$ is curl free. 
As can be observed, the time rates of $\boldsymbol{p}, \boldsymbol{G}$ and $j$, present in the left hand side of the system of conservation laws, are also involved in the stabilisation terms $\boldsymbol{p}_{\boldsymbol{G}}^{s t}, \boldsymbol{p}_{j}^{s t}, \boldsymbol{G}^{s t}$ and $j^{s t}$, resulting in an implicit formulation, which could lead to a costly algorithm. To reduce the level of implicitness of the formulation, expressions (23a-23b) can be further enhanced by adding the corresponding time integrated stabilisation terms $\int_{t} \tau_{\boldsymbol{G} \boldsymbol{p}} \boldsymbol{\mathcal { R }}_{\boldsymbol{G}} d t$ and $\int_{t} \tau_{j \boldsymbol{p}} \mathcal{R}_{j} d t$ for $\boldsymbol{G}^{s t}$ and $j^{s t}$, respectively, resulting in new expressions for $\boldsymbol{G}^{s t}$ and $j^{s t}$ as follows

$$
\begin{aligned}
\boldsymbol{G}^{s t} & :=\boldsymbol{G}+\tau_{\boldsymbol{G} \boldsymbol{p}} \boldsymbol{\mathcal { R }}_{\boldsymbol{G}}+\alpha(\boldsymbol{\nabla} \boldsymbol{u}-\boldsymbol{G}), \\
j^{s t} & :=j+\tau_{j \boldsymbol{p}} \mathcal{R}_{j}+\beta(\operatorname{div} \boldsymbol{u}-j)
\end{aligned}
$$

where $\alpha$ (already introduced in reference [44]) and $\beta$ are non-dimensional stabilisation parameters in the range 0 to 0.5 . In order to develop a formulation consistent with that of a fractional step approach (refer to section 2.4.1), it is interesting to re-scale the stabilisation coefficients $\tau_{j p}$ and $\beta$ in $(27 \mathrm{~b})$, by means of the dimensionless ratio $\frac{\mu}{\kappa}$ as

$$
j^{s t}:=j+\tau_{j p} \frac{\mu}{\kappa} \mathcal{R}_{j}+\beta \frac{\mu}{\kappa}(\operatorname{div} \boldsymbol{u}-j) .
$$

Note that all stabilising terms $(26 \mathrm{a}-26 \mathrm{~b})$ and $(27 \mathrm{a}, 28)$ are weighted residuals, ensuring the consistency of the numerical formulation and thus preserving the order of convergence. For the examples shown in this paper, the formulation was simplified by using $\tau_{j p}=\tau_{p G}=0$. With this consideration, the system of equations (24) and (25a-25b) becomes fully decoupled, enabling its resolution in a sequential manner. Equation (25a) is first solved for $\dot{\boldsymbol{G}}$ which can then be substituted into (24) to yield $\dot{\boldsymbol{p}}$. Once $\dot{\boldsymbol{p}}$ is known, $\dot{j}$ can then be determined from equation (25b) via prior evaluation of $\boldsymbol{p}_{j}^{s t}$. On the contrary, if either $\tau_{p G}$ or $\tau_{j p}$ are non-zero, an iterative procedure is then required.

Finally, the stabilised semidiscrete nodal equations which have been produced can then be explicitly integrated from time step $t^{n}$ to $t^{n+1}$. In this case, the explicit one-step two-stage Total Variation Diminishing RungeKutta (TVD-RK) time integrator [72] is preferred due to its excellent TVD properties (refer to Section 4 in [44] for further discussion). The evaluation of the maximum time increment $\Delta t$ is intimately related to the minimum size of element $h_{\min }$ and the maximum wave speed $c_{\max }=c_{p}$ (15) via the Courant-Friedrichs-Lewy number $\alpha_{C F L}[51]$. 


\subsection{Fractional step approach: small deformations}

As it is well known, in the case of fully incompressible or nearly incompressible materials, the volumetric wave speed $c_{p}$ can reach very large values leading to prohibitively small time steps [51]. This can have a very negative effect in the computational efficiency of the algorithm. A popular approach to handle incompressibility in explicit schemes is the fractional step method, originally developed in [52-54]. This methodology can be adapted to our mixed formulation (1a-1c) in order to alleviate the numerical difficulties associated with the existence of a saddle-point.

The conservation equation for the volumetric strain (1c) is replaced by the constraint $\operatorname{div}\left(\frac{\boldsymbol{p}}{\rho}\right)=0$. As presented at the end of section 2.2 , the problem is then dominated by shear waves of speed $c_{s}$. In this case, the new unknowns for the problem are $\{\boldsymbol{p}, \boldsymbol{G}, p\}$ ( $j$ is replaced with $p$ ), as the volumetric strain $j$ is constrained to be always zero throughout the entire deformation process.

The time update of the linear momentum from $\boldsymbol{p}^{n}$ to $\boldsymbol{p}^{n+1}$ over a time step $\Delta t$ is split in two stages. Firstly, the algorithm is advanced explicitly yielding an intermediate linear momentum $\boldsymbol{p}^{\text {int }}$ which is then projected after implicitly solving a Poisson-like equation [73] (also known as pressure correction). In this approach, it is traditional [74] to first discretise in time and then discretise in space (i.e. using a suitable PG stabilisation). Therefore, the first (predictor or intermediate) step of the scheme is defined as

$$
\begin{aligned}
\frac{\left(\boldsymbol{p}^{i n t}-\boldsymbol{p}^{n}\right)}{\Delta t}-\operatorname{div} \boldsymbol{\sigma}^{\operatorname{dev}, n}-\nabla p^{n}-\rho \boldsymbol{b}^{n} & =\mathbf{0}, \\
\frac{\boldsymbol{G}^{n+1}-\boldsymbol{G}^{n}}{\Delta t}-\boldsymbol{\nabla}\left(\frac{\boldsymbol{p}^{n}}{\rho}\right) & =\mathbf{0},
\end{aligned}
$$

where $\boldsymbol{p}^{\text {int }}$ stands for the linear momentum at an intermediate stage and the second (corrector or projection) step becomes

$$
\frac{\left(\boldsymbol{p}^{n+1}-\boldsymbol{p}^{i n t}\right)}{\Delta t}-\nabla\left(p^{n+1}-p^{n}\right)=\mathbf{0} .
$$

The summation of both equations (29a) and (30) recovers the original assumption in which the pressure variable is treated implicitly in the formulation. Application of the divergence operator to (30) produces

$$
\operatorname{div}\left(\frac{\boldsymbol{p}^{n+1}}{\rho}\right)-\operatorname{div}\left(\frac{\boldsymbol{p}^{i n t}}{\rho}\right)-\frac{\Delta t}{\rho} \nabla^{2}\left(p^{n+1}-p^{n}\right)=0
$$


where the Laplacian operator $\nabla^{2}(\cdot)=\operatorname{div} \nabla(\cdot)$ has been introduced. For simplicity and without loss of generality, the density $\rho$ has been assumed to be constant across the entire domain. To allow for the case of nearly incompressible deformation, the first term in (31) can be written as follows

$$
\operatorname{div}\left(\frac{\boldsymbol{p}^{n+1}}{\rho}\right)=\frac{p^{n+1}-p^{n}}{\kappa \Delta t}
$$

For a truly incompressible material, $\kappa=\infty$ and the right hand side of equation (32) vanishes, resulting in the incompressibility constraint. Substituting (32) into (31) renders

$$
\frac{1}{\kappa \Delta t}\left(p^{n+1}-p^{n}\right)-\operatorname{div}\left(\frac{\boldsymbol{p}^{i n t}}{\rho}\right)-\frac{\Delta t}{\rho} \nabla^{2}\left(p^{n+1}-p^{n}\right)=0 .
$$

In the following section, the weak statements for (29a-29b) and (33) are derived by employing suitable Petrov-Galerkin stabilisations. The linear momentum can finally be updated (30) using a classical Bubnov-Galerkin approach via the pressure correction $p^{n+1}-p^{n}$ once (33) has been solved.

\subsubsection{Variational linearised fractional step formulation}

To obtain a variational statement for the fractional step formulation, we first need to define the corresponding residuals of equations (29a), (29b) and (33) as,

$$
\begin{aligned}
\mathcal{R}_{\boldsymbol{p}^{i n t}} & :=\operatorname{div} \boldsymbol{\sigma}^{\operatorname{dev}, n}+\nabla p^{n}+\rho \boldsymbol{b}^{n}-\frac{\left(\boldsymbol{p}^{i n t}-\boldsymbol{p}^{n}\right)}{\Delta t}, \\
\mathcal{R}_{\boldsymbol{G}} & :=\boldsymbol{\nabla}\left(\frac{\boldsymbol{p}^{n}}{\rho}\right)-\frac{\boldsymbol{G}^{n+1}-\boldsymbol{G}^{n}}{\Delta t} \\
\mathcal{R}_{j} & :=\operatorname{div}\left(\frac{\boldsymbol{p}^{i n t}}{\rho}\right)+\frac{\Delta t}{\rho} \nabla^{2}\left(p^{n+1}-p^{n}\right)-\frac{1}{\kappa \Delta t}\left(p^{n+1}-p^{n}\right), \\
\mathcal{R}_{\boldsymbol{p}} & :=\boldsymbol{\nabla} p^{n+1}-\frac{\left(\boldsymbol{p}^{n+1}-\boldsymbol{p}^{i n t}\right)}{\Delta t} .
\end{aligned}
$$

Using appropriately stabilised conjugate virtual fields $\delta \mathcal{V}^{s t}=\left[\delta \boldsymbol{v}^{s t}, \delta \boldsymbol{\sigma}^{s t}, \delta q^{s t}\right]^{T}$, already defined in (19a-19c), a variational statement is defined as

$$
\delta W^{\mathrm{PG}}=\int_{v} \delta \boldsymbol{v}^{s t} \cdot \mathcal{R}_{p^{i n t}} d v+\int_{v} \delta \boldsymbol{\sigma}^{s t}: \boldsymbol{\mathcal { R }}_{\boldsymbol{G}} d v+\int_{v} \delta q^{s t} \mathcal{R}_{j} d v=0 .
$$


Following a similar finite element spatial discretisation strategy as that presented previously, where $\{\boldsymbol{p}, \boldsymbol{G}, p\}$ and $\left\{\delta \boldsymbol{v}^{s t}, \delta \boldsymbol{\sigma}^{s t}, \delta q^{s t}\right\}$ are expanded in terms of nodal values and corresponding linear shape functions, the resulting predictor system of equations yields

$$
\begin{aligned}
\sum_{b} \mathcal{M}_{a b} \frac{\left(\boldsymbol{p}_{b}^{i n t}-\boldsymbol{p}_{b}^{n}\right)}{\Delta t} & =\int_{\partial v} N_{a} \boldsymbol{t}^{B, n} d a+\int_{v} N_{a} \rho \boldsymbol{b}^{n} d v-\int_{v} \boldsymbol{\sigma}\left(\boldsymbol{G}^{s t}, p^{s t}\right) \nabla N_{a} d v \\
\sum_{b} \mathcal{M}_{a b} \frac{\left(\boldsymbol{G}_{b}^{n+1}-\boldsymbol{G}_{b}^{n}\right)}{\Delta t} & =\int_{\partial v} N_{a}\left(\frac{\boldsymbol{p}^{B, n}}{\rho} \otimes \boldsymbol{n}\right) d a-\int_{v} \frac{\boldsymbol{p}_{\boldsymbol{G}}^{s t}}{\rho} \otimes \nabla N_{a} d v
\end{aligned}
$$

where

$$
\begin{aligned}
\boldsymbol{G}^{s t} & :=\boldsymbol{G}^{n}+\tau_{\boldsymbol{G} \boldsymbol{p}} \boldsymbol{\mathcal { R }}_{\boldsymbol{G}}+\alpha\left(\boldsymbol{\nabla} \boldsymbol{u}^{n}-\boldsymbol{G}^{n}\right) \\
p^{s t} & :=p^{n}+\tau_{j \boldsymbol{p}} \mu \mathcal{R}_{j}+\beta \mu\left(\operatorname{div} \boldsymbol{u}^{n}-\frac{p^{n}}{\kappa}\right) \\
\boldsymbol{p}_{\boldsymbol{G}}^{s t} & :=\boldsymbol{p}^{n}+\tau_{\boldsymbol{p} \boldsymbol{G}} \boldsymbol{\mathcal { R }}_{\boldsymbol{p}^{i n t}} .
\end{aligned}
$$

Notice that for the case of using linear shape functions for the expansion of the pressure field, the second term (Laplacian term) on the right side of (34c) vanishes. It is interesting to note how equations (28) and (37b) are related, as multiplication of equation (28) by $\kappa$ yields (37b). For the case of incompressible materials, where $\kappa=\infty$, the term in parenthesis on the right hand side of (37b) yields the volumetric constraint.

As discussed in Section 2.3, by setting $\tau_{j \boldsymbol{p}}=\tau_{\boldsymbol{p} \boldsymbol{G}}=0$, equations (36a$36 \mathrm{~b}$ ) can be solved sequentially. Finally, the corrector system of equations emerges as

$$
\sum_{b}\left[\mathcal{M}_{a b}^{v o l}+\frac{\Delta t^{2}}{\rho} \mathcal{K}_{a b}\right]\left(\frac{p_{b}^{n+1}-p_{b}^{n}}{\Delta t}\right) d v=\int_{\partial v} N_{a}\left(\frac{\boldsymbol{p}^{B}}{\rho} \cdot \boldsymbol{n}\right) d a-\int_{v} \frac{\boldsymbol{p}_{j}^{s t}}{\rho} \cdot \nabla N_{a} d v
$$

where the mass matrix contribution $\mathcal{M}_{a b}^{\text {vol }}$, the viscosity matrix contribution 
$\mathcal{K}_{a b}$ and the stabilised linear momentum $\boldsymbol{p}_{j}^{s t}$ are defined as

$$
\begin{aligned}
\mathcal{M}_{a b}^{v o l} & :=\int_{v} \frac{1}{\kappa} N_{a} N_{b} d v, \\
\mathcal{K}_{a b} & :=\int_{v} \boldsymbol{\nabla} N_{a} \cdot \boldsymbol{\nabla} N_{b} d v, \\
\boldsymbol{p}_{j}^{s t} & :=\boldsymbol{p}^{\text {int }}+\tau_{\boldsymbol{p} j} \boldsymbol{\mathcal { R }}_{\boldsymbol{p}^{i n t}},
\end{aligned}
$$

respectively. Note that equation $(25 \mathrm{~b})$ can be identified as a particular case of (38) by neglecting the viscous contribution $\mathcal{K}_{a b}$. For the case of full incompressibility $(\kappa=\infty)$, the mass matrix contribution vanishes. Once the pressure increment (38) is known, we can subsequently update the linear momentum $\boldsymbol{p}^{n+1}$ using a standard Bubnov-Galerkin formulation $\left(\delta W^{\mathrm{BG}}=\right.$ $\left.\int_{v} \delta \boldsymbol{v} \cdot \boldsymbol{\mathcal { R }}_{\boldsymbol{p}} d v=0\right)$ to give

$$
\sum_{b} \mathcal{M}_{a b} \frac{\boldsymbol{p}_{b}^{n+1}-\boldsymbol{p}_{b}^{\text {int }}}{\Delta t}=\int_{v} N_{a} \boldsymbol{\nabla}\left(p^{n+1}-p^{n}\right) d v .
$$

As mentioned at the end of section 2.3, the scheme is explicitly driven in time via a TVD-RK time integrator where, in this case, the maximum allowable time step is controlled by the shear wave speed $c_{s}$.

\section{Nonlinear reversible elastodynamics}

\subsection{Enhanced $\boldsymbol{p}-\boldsymbol{F}-J$ mixed methodology}

Let us consider the motion of a continuum which in its initial or material configuration is defined by a domain $V \subset \mathbb{R}^{3}$ of boundary $\partial V$ with outward unit normal $\boldsymbol{N}$. After the motion, the continuum occupies a spatial configuration defined by a domain $v \subset \mathbb{R}^{3}$ of boundary $\partial v$ with outward unit normal $\boldsymbol{n}$. The motion is defined by a time $t$ dependent mapping field $\boldsymbol{\phi}$ which links a material particle from material configuration $\boldsymbol{X} \in V$ to spatial configuration $\boldsymbol{x} \in v$ according to $\boldsymbol{x}=\boldsymbol{\phi}(\boldsymbol{X}, t)$. It is possible to define the motion through a system of first order conservation laws expressed in a Lagrangian 
format as follows

$$
\begin{aligned}
\frac{\partial \boldsymbol{p}}{\partial t}-\mathrm{DIV} \boldsymbol{P} & =\rho_{0} \boldsymbol{b} \\
\frac{\partial \boldsymbol{F}}{\partial t}-\mathrm{DIV}\left(\frac{1}{\rho_{0}} \boldsymbol{p} \otimes \boldsymbol{I}\right) & =\mathbf{0}, \\
\frac{\partial J}{\partial t}-\mathrm{DIV}\left(\boldsymbol{H}_{\boldsymbol{F}}^{T} \frac{\boldsymbol{p}}{\rho}\right) & =0, \quad \boldsymbol{H}_{\boldsymbol{F}}:=J_{\boldsymbol{F}} \boldsymbol{F}^{-T}, \quad J_{\boldsymbol{F}}=\operatorname{det} \boldsymbol{F}, \\
\frac{\partial E_{T}}{\partial t}-\mathrm{DIV}\left(\frac{1}{\rho} \boldsymbol{P}^{T} \boldsymbol{p}-\boldsymbol{Q}\right) & =s .
\end{aligned}
$$

Equation (41a) represents the conservation of linear momentum $\boldsymbol{p}$, where $\rho_{0}$ is the density of the continuum in the initial configuration, $\boldsymbol{b}$ is a body force per unit of mass, $\boldsymbol{P}$ is the first Piola-Kirchhoff stress tensor and DIV is the divergence operator in the material configuration. Equation (41b) is a conservation equation for the deformation gradient tensor $\boldsymbol{F}$ whilst (41c) is a conservation equation for the Jacobian $J$ of the deformation where $\boldsymbol{H}_{\boldsymbol{F}}^{T}$ is defined as the cofactor of the deformation. Finally, equation (41d) represents the conservation of the total energy per unit of undeformed volume $E_{T}$ with $\boldsymbol{Q}$ the heat flux and $s$ the heat source.

Analogously to section 2.1, in the case of adiabatic isothermal deformations, this last equation (41d) is fully decoupled from the rest of the system. In addition, the stress $\boldsymbol{P}$ is considered a function of $\boldsymbol{F}$ and $J$, which are evolved in time through equations (41b) and (41c), respectively. Again, notice that in this mixed $\boldsymbol{p}-\boldsymbol{F}$ - $J$ formulation, $\boldsymbol{F}$ and $J$ are not computed from the mapping $\phi$, namely $\nabla_{0} \phi$ and $\operatorname{det} \boldsymbol{\nabla}_{0} \boldsymbol{\phi}$, respectively ${ }^{4}$.

In order to close the coupled system defined by (41a-41c), the underlying conservation laws have to be supplemented with an appropriate constitutive model obeying both the laws of thermodynamics and the principle of objectivity [61, 75-77]. One of the simplest models satisfying the above conditions is the well-known hyperelastic nearly incompressible Neo-Hookean (NH) model. Its strain energy functional $\psi(\boldsymbol{F}, J)$ can be additively decomposed into a deviatoric contribution $\psi^{\operatorname{dev}}\left(J_{\boldsymbol{F}}^{-1 / 3} \boldsymbol{F}\right)$ and a volumetric contribution $\psi^{\text {vol }}(J)$ defined by

$$
\psi^{d e v}=\frac{1}{2} \mu\left(J_{\boldsymbol{F}}^{-2 / 3}(\boldsymbol{F}: \boldsymbol{F})-3\right), \quad \psi^{v o l}=\frac{1}{2} \kappa(J-1)^{2},
$$

\footnotetext{
${ }^{4} \nabla_{0}$ represents the gradient operator with respect to the material configuration
} 
where $\kappa$ and $\mu$ are the bulk and shear moduli, respectively. The corresponding first Piola-Kirchhoff stress tensor $\boldsymbol{P}$ can then be derived as

$$
\boldsymbol{P}=\boldsymbol{P}^{d e v}+\boldsymbol{P}^{v o l}, \quad \boldsymbol{P}^{d e v}=\frac{\partial \psi^{d e v}}{\partial \boldsymbol{F}}, \quad \boldsymbol{P}^{v o l}=\frac{d \psi^{v o l}}{d J} \boldsymbol{H}_{\boldsymbol{F}}=p \boldsymbol{H}_{\boldsymbol{F}},
$$

where the deviatoric contribution of $\boldsymbol{P}$ and the hydrostatic pressure $p$ are

$$
\boldsymbol{P}^{d e v}=\mu J_{\boldsymbol{F}}^{-2 / 3}\left(\boldsymbol{F}-\frac{1}{3}(\boldsymbol{F}: \boldsymbol{F}) \boldsymbol{F}^{-T}\right), \quad p=\kappa(J-1) .
$$

The system of conservation laws (41a-41c) can be recast in a more compact manner as

$$
\frac{\partial \mathcal{U}}{\partial t}+\frac{\partial \mathcal{F}_{I}}{\partial X_{I}}=\mathcal{S}, \quad I=1,2,3
$$

where $\mathcal{U}$ is the vector of conservation variables, $\mathcal{F}_{I}$ denotes the flux vector in the spatial direction $I$ and $\mathcal{S}$ the source term, namely

$$
\mathcal{U}=\left[\begin{array}{c}
\boldsymbol{p} \\
\boldsymbol{F} \\
J
\end{array}\right], \quad \mathcal{F}_{\boldsymbol{N}}=\mathcal{F}_{I} N_{I}=\left[\begin{array}{c}
-\boldsymbol{P} \boldsymbol{N} \\
\frac{-1}{\rho_{0}} \boldsymbol{p} \otimes \boldsymbol{N} \\
\frac{-1}{\rho_{0}} \boldsymbol{p} \cdot \boldsymbol{H}_{\boldsymbol{F}} \boldsymbol{N}
\end{array}\right], \quad \mathcal{S}=\left[\begin{array}{c}
\rho_{0} \boldsymbol{b} \\
\mathbf{0} \\
0
\end{array}\right]
$$

Similarly to section 2.1, the above system can be rewritten in quasi-linear format as

$$
\frac{\partial \mathcal{U}}{\partial t}+\mathcal{A}_{I} \frac{\partial \mathcal{U}}{\partial X_{I}}=\mathcal{S}, \quad I=1,2,3,
$$

where the corresponding flux Jacobian matrix $\mathcal{A}_{N}=\mathcal{A}_{I} N_{I}$ is

$$
\boldsymbol{A}_{\boldsymbol{N}}=\left[\begin{array}{ccc}
-\frac{\partial(\boldsymbol{P} \boldsymbol{N})}{\partial \boldsymbol{p}} & -\frac{\partial(\boldsymbol{P} \boldsymbol{N})}{\partial \boldsymbol{F}} & -\frac{\partial(\boldsymbol{P} \boldsymbol{N})}{\partial J} \\
-\frac{\partial\left(\frac{1}{\rho_{0}} \boldsymbol{p} \otimes \boldsymbol{N}\right)}{\partial \boldsymbol{p}} & -\frac{\partial\left(\frac{1}{\rho_{0}} \boldsymbol{p} \otimes \boldsymbol{N}\right)}{\partial \boldsymbol{F}} & -\frac{\partial\left(\frac{1}{\rho_{0}} \boldsymbol{p} \otimes \boldsymbol{N}\right)}{\partial J} \\
-\frac{\partial\left(\frac{1}{\rho_{0}} \boldsymbol{p} \cdot \boldsymbol{H}_{\boldsymbol{F}} \boldsymbol{N}\right)}{\partial \boldsymbol{p}} & -\frac{\partial\left(\frac{1}{\rho_{0}} \boldsymbol{p} \cdot \boldsymbol{H}_{\boldsymbol{F}} \boldsymbol{N}\right)}{\partial \boldsymbol{F}} & -\frac{\partial\left(\frac{1}{\rho_{0}} \boldsymbol{p} \cdot \boldsymbol{H}_{\boldsymbol{F}} \boldsymbol{N}\right)}{\partial J}
\end{array}\right] .
$$

Substitution of (43-44) into (48) yields

$$
\mathcal{A}_{\boldsymbol{N}}=\left[\begin{array}{ccc}
\boldsymbol{0}_{3 \times 3} & -\mathcal{C}_{\boldsymbol{N}} & -\kappa \boldsymbol{H}_{\boldsymbol{F}} \boldsymbol{N} \\
-\frac{1}{\rho_{0}} \boldsymbol{I}_{\boldsymbol{N}} & \boldsymbol{0}_{3 \times 3 \times 3 \times 3} & \mathbf{0}_{3 \times 3 \times 1} \\
-\frac{1}{\rho_{0}} \boldsymbol{N} & \mathbf{0}_{3 \times 3} & 0
\end{array}\right]
$$

where

$$
\begin{aligned}
{\left[\mathcal{C}_{N}\right]_{i j J} } & =[\mathcal{C}]_{i I j J} N_{I}, \quad[\mathcal{C}]_{i I j J}=\frac{\partial[\boldsymbol{P}]_{i I}}{\partial[\boldsymbol{F}]_{j J}} \\
{\left[\boldsymbol{I}_{\boldsymbol{N}}\right]_{i J k} } & =\delta_{i k} N_{J}
\end{aligned}
$$




\subsection{Eigenvalue structure}

Apart from objectivity and compliance with the second law of thermodynamics, the constitutive model under consideration must also satisfy another requirement, namely rank one convexity, also known as the Legendre and Hadamard condition [58]. Satisfaction of this condition is equivalent to the existence of travelling waves with real wave speeds. Hence, the study of the eigenvalue structure of the system of conservation laws becomes of paramount importance. The eigenvalues and eigenvectors of system (47) can be evaluated considering plane wave solutions of the form

$$
\mathcal{U}=\phi\left(\boldsymbol{X} \cdot \boldsymbol{N}-c_{\alpha} t\right) \overline{\mathcal{U}}_{\alpha}=\phi\left(\boldsymbol{X} \cdot \boldsymbol{N}-c_{\alpha} t\right)\left[\begin{array}{c}
\boldsymbol{p}_{\alpha} \\
\boldsymbol{F}_{\alpha} \\
J_{\alpha}
\end{array}\right]
$$

where $c_{\alpha}$ are the wave speeds corresponding to the eigenvector $\overline{\mathcal{U}}_{\alpha}$. The resulting eigen-system $c_{\alpha} \overline{\mathcal{U}}_{\alpha}=\mathcal{A}_{N} \overline{\mathcal{U}}_{\alpha}$ is of the form

$$
\begin{aligned}
c_{\alpha} \boldsymbol{p}_{\alpha} & =-\mathcal{C}_{\boldsymbol{N}}: \boldsymbol{F}_{\alpha}-\kappa \boldsymbol{H}_{\boldsymbol{F}} J_{\alpha} \boldsymbol{N} \\
c_{\alpha} \boldsymbol{F}_{\alpha} & =-\frac{1}{\rho_{0}} \boldsymbol{p}_{\alpha} \otimes \boldsymbol{N} \\
c_{\alpha} J_{\alpha} & =-\frac{1}{\rho_{0}} \boldsymbol{p}_{\alpha} \cdot \boldsymbol{H}_{\boldsymbol{F}} \boldsymbol{N}-\frac{1}{\rho_{0}} \boldsymbol{p} \cdot\left(\frac{\partial \boldsymbol{H}_{\boldsymbol{F}}}{\partial \boldsymbol{F}}: \boldsymbol{F}_{\alpha}\right) \boldsymbol{N} .
\end{aligned}
$$

Substitution of equation (52b) into (52c) yields

$$
c_{\alpha} J_{\alpha}=-\frac{1}{\rho_{0}} \boldsymbol{p}_{\alpha} \cdot \boldsymbol{H}_{\boldsymbol{F}} \boldsymbol{N}+\frac{1}{\rho_{0}^{2} c_{\alpha}}(\boldsymbol{p} \otimes \boldsymbol{N}): \frac{\partial \boldsymbol{H}_{\boldsymbol{F}}}{\partial \boldsymbol{F}}:\left(\boldsymbol{p}_{\alpha} \otimes \boldsymbol{N}\right) .
$$

It is possible to demonstrate after some algebra that the last term in the right hand side of above equation (53) is zero, yielding the reduced equation

$$
c_{\alpha} J_{\alpha}=-\frac{1}{\rho_{0}} \boldsymbol{p}_{\alpha} \cdot \boldsymbol{H}_{\boldsymbol{F}} \boldsymbol{N}
$$

Substitution of equations (52b) and (54) into (52a) results in

$$
\rho_{0} c_{\alpha}^{2} \boldsymbol{p}_{\alpha}=\left[\mathcal{C}_{\boldsymbol{N} \boldsymbol{N}}+\kappa\left(\boldsymbol{H}_{\boldsymbol{F}} \boldsymbol{N}\right) \otimes\left(\boldsymbol{H}_{\boldsymbol{F}} \boldsymbol{N}\right)\right] \boldsymbol{p}_{\alpha}, \quad\left[\mathcal{C}_{\boldsymbol{N} N}\right]_{i j}=[\mathcal{C}]_{i I j J} N_{I} N_{J}
$$


The tensor $\mathcal{C}_{\boldsymbol{N} \boldsymbol{N}}$ for the constitutive model under consideration (42) can be shown to be

$$
\begin{aligned}
\mathcal{C}_{\boldsymbol{N} N}= & -\frac{2}{3} \mu J_{\boldsymbol{F}}^{-5 / 3}\left(\left(\boldsymbol{H}_{\boldsymbol{F}} \boldsymbol{N}\right) \otimes(\boldsymbol{F} \boldsymbol{N})+(\boldsymbol{F} \boldsymbol{N}) \otimes\left(\boldsymbol{H}_{\boldsymbol{F}} \boldsymbol{N}\right)\right) \\
& +\mu J_{\boldsymbol{F}}^{-2 / 3} \boldsymbol{I}+\frac{5}{9} \mu J_{\boldsymbol{F}}^{-8 / 3}(\boldsymbol{F}: \boldsymbol{F})\left(\boldsymbol{H}_{\boldsymbol{F}} \boldsymbol{N}\right) \otimes\left(\boldsymbol{H}_{\boldsymbol{F}} \boldsymbol{N}\right) .
\end{aligned}
$$

Combining equations (55) and (56) and introducing the vectors $\boldsymbol{m}:=\boldsymbol{H}_{\boldsymbol{F}} \boldsymbol{N}$ and $\boldsymbol{m}^{*}:=\boldsymbol{F} \boldsymbol{N}$, it yields

$$
\rho_{0} c_{\alpha}^{2} \boldsymbol{p}_{\alpha}=\left[\gamma_{1}\left(\boldsymbol{m} \otimes \boldsymbol{m}^{*}+\boldsymbol{m}^{*} \otimes \boldsymbol{m}\right)+\gamma_{2} \boldsymbol{I}+\gamma_{3} \boldsymbol{m} \otimes \boldsymbol{m}\right] \boldsymbol{p}_{\alpha},
$$

where

$$
\begin{aligned}
\gamma_{1} & =-\frac{2}{3} \mu J_{\boldsymbol{F}}^{-5 / 3}, \\
\gamma_{2} & =\mu J_{\boldsymbol{F}}^{-2 / 3}, \\
\gamma_{3} & =\kappa+\frac{5}{9} \mu J_{\boldsymbol{F}}^{-8 / 3}(\boldsymbol{F}: \boldsymbol{F}) .
\end{aligned}
$$

Although it is possible to obtain a closed form solution for the eigen-system defined above, it is sufficient to compute bounds of the wave speeds. This scenario arises when the vectors $\boldsymbol{m}$ and $\boldsymbol{m}^{*}$ are co-linear, which is attained when $\boldsymbol{N}$ is a principal direction. In this particular case, it is easy to prove that $\boldsymbol{m}^{*}=\lambda \boldsymbol{n}$ and $\boldsymbol{m}=\frac{J_{\boldsymbol{F}}}{\lambda} \boldsymbol{n}$ where $\lambda$ is the stretch in the spatial direction $\boldsymbol{n}$ and the system (57) yields

$$
\rho_{0} c_{\alpha}^{2} \boldsymbol{p}_{\alpha}=\left[\left(2 \gamma_{1}\left(\frac{J_{\boldsymbol{F}}}{\lambda}\right)+\gamma_{3}\left(\frac{J_{\boldsymbol{F}}}{\lambda}\right)^{2}\right) \boldsymbol{n} \otimes \boldsymbol{n}+\gamma_{2} \boldsymbol{I}\right] \boldsymbol{p}_{\alpha} .
$$

It can be seen that the system contains three pairs of non-zero eigenvalues corresponding to the volumetric and shear waves as

$c_{1,2}= \pm c_{p}, c_{p}=\sqrt{\frac{\left(2 \gamma_{1}\left(\frac{J_{F}}{\lambda}\right)+\gamma_{3}\left(\frac{J_{F}}{\lambda}\right)^{2}+\gamma_{2}\right)}{\rho_{0}}}, \quad c_{3,4}=c_{5,6}= \pm c_{s}, c_{s}=\sqrt{\frac{\gamma_{2}}{\rho_{0}}}$,

with eigenvectors

$\overline{\mathcal{U}}_{1,2}=\left[\begin{array}{c}\boldsymbol{n} \\ \pm \frac{1}{\rho_{0} c_{p}} \boldsymbol{n} \otimes \boldsymbol{N} \\ \pm \frac{1}{\rho_{0} c_{p}} \frac{J_{\boldsymbol{F}}}{\lambda}\end{array}\right], \quad \overline{\mathcal{U}}_{3,4}=\left[\begin{array}{c}\boldsymbol{t}_{1} \\ \pm \frac{1}{\rho_{0} c_{s}} \boldsymbol{t}_{1} \otimes \boldsymbol{N} \\ 0\end{array}\right], \quad \overline{\mathcal{U}}_{5,6}=\left[\begin{array}{c}\boldsymbol{t}_{2} \\ \pm \frac{1}{\rho_{0} c_{s}} \boldsymbol{t}_{2} \otimes \boldsymbol{N} \\ 0\end{array}\right]$, 
where $\boldsymbol{t}_{1}$ and $\boldsymbol{t}_{2}$ are two arbitrary tangential vectors orthogonal to $\boldsymbol{n}$. The rest of the eigenvalues are zero and have null associated velocity components.

To ensure the hyperbolicity of the problem, $c_{p}$ and $c_{s}$ must be real. This is easy to prove noticing that $\gamma_{2} \geq 0$ ( $c_{s}$ are real waves) and

$2 \gamma_{1}\left(\frac{J_{\boldsymbol{F}}}{\lambda}\right)+\gamma_{3}\left(\frac{J_{\boldsymbol{F}}}{\lambda}\right)^{2}+\gamma_{2}=\kappa\left(\frac{J_{\boldsymbol{F}}}{\lambda}\right)^{2}+\mu J_{\boldsymbol{F}}^{-2 / 3}\left(1+\frac{5}{9} \frac{\boldsymbol{F}: \boldsymbol{F}}{\lambda^{2}}-\frac{4}{3 \lambda}\right) \geq 0$,

proving that $c_{p}$ are also real waves. This proves the rank one convexity of the strain energy potential and guarantees the existence of physical waves propagating throughout the domain [58].

For a fully incompressible material, the conservation equation for the volumetric strain (41c) is replaced by the constraint $\boldsymbol{H}_{\boldsymbol{F}}: \boldsymbol{\nabla}_{0}\left(\frac{\boldsymbol{p}}{\rho_{0}}\right)=0$ and thus, equation (54) gets replaced by the constraint $\boldsymbol{p}_{\alpha} \cdot \boldsymbol{H}_{\boldsymbol{F}} \boldsymbol{N}=0$. This constraint, once substituted into equation (57) yields

$$
\rho_{0} c_{\alpha}^{2} \boldsymbol{p}_{\alpha}=\gamma_{2} \boldsymbol{p}_{\alpha}
$$

leading to only two pairs of non-zero eigenvalues corresponding to the shear waves $c_{3,4}=c_{5,6}= \pm c_{s}$ with eigenvectors $\overline{\mathcal{U}}_{3,4}$ and $\overline{\mathcal{U}}_{5,6}$.

\subsection{Nonlinear Petrov-Galerkin formulation}

The stabilised variational statement for reversible nonlinear elastodynamics can also be derived through the use of work conjugate principles [44]. To achieve this, we need to define appropriate stabilised conjugate variables $\delta \mathcal{V}^{s t}=\left[\delta \boldsymbol{v}^{s t}, \delta \boldsymbol{P}^{s t}, \delta q^{s t}\right]^{T}$ via (18) using the appropriate flux Jacobian matrix $\mathcal{A}_{I}$ as

$$
\begin{aligned}
\delta \boldsymbol{v}^{s t} & =\delta \boldsymbol{v}-\frac{\tau_{\boldsymbol{p} \boldsymbol{F}}}{\rho_{0}} \operatorname{DIV} \delta \boldsymbol{P}-\frac{\tau_{\boldsymbol{p} J}}{\rho_{0}} \boldsymbol{H}_{\boldsymbol{F}} \boldsymbol{\nabla}_{0} \delta q \\
\delta \boldsymbol{P}^{s t} & =\delta \boldsymbol{P}-\tau_{\boldsymbol{F} \boldsymbol{p}} \mathcal{C}: \boldsymbol{\nabla}_{0} \delta \boldsymbol{v}-\tau_{\boldsymbol{F} J}\left(\frac{\boldsymbol{p}}{\rho_{0}} \otimes \boldsymbol{\nabla}_{0} \delta q\right): \frac{\partial \boldsymbol{H}_{\boldsymbol{F}}}{\partial \boldsymbol{F}} \\
\delta q^{s t} & =\delta q-\tau_{J \boldsymbol{p}} \kappa \boldsymbol{H}_{\boldsymbol{F}}: \boldsymbol{\nabla}_{0} \delta \boldsymbol{v}
\end{aligned}
$$


and the residuals $\mathcal{R}=\left[\boldsymbol{\mathcal { R }}_{\boldsymbol{v}}, \boldsymbol{\mathcal { R }}_{\boldsymbol{F}}, \mathcal{R}_{J}\right]^{T}$ of the conservation laws (41a-41c) are defined by

$$
\begin{aligned}
& \mathcal{R}_{\boldsymbol{p}}=\operatorname{DIV} \boldsymbol{P}+\rho_{0} \boldsymbol{b}-\dot{\boldsymbol{p}} \\
& \boldsymbol{\mathcal { R }}_{\boldsymbol{F}}=\nabla_{0}\left(\frac{\boldsymbol{p}}{\rho_{0}}\right)-\dot{\boldsymbol{F}} \\
& \mathcal{R}_{J}=\operatorname{DIV}\left(\boldsymbol{H}_{\boldsymbol{F}}{ }^{T} \frac{\boldsymbol{p}}{\rho_{0}}\right)-\dot{J}
\end{aligned}
$$

Using expressions in (64a-64c) and (65a-65c), it is now possible to derive the stabilised weak statement by multiplying appropriate conjugate virtual fields $\delta \mathcal{V}^{s t}$ with the corresponding residuals $\mathcal{R}$ and integrating over the volume $\mathrm{V}$, to give

$$
\delta W^{\mathrm{PG}}=\int_{V} \delta \mathcal{V}^{s t} \cdot \mathcal{R} d V=0 .
$$

Following an identical finite element methodology as that outlined in Section 2.3, the conjugate virtual fields $\left\{\delta \boldsymbol{v}^{s t}, \delta \boldsymbol{P}^{s t}, \delta q^{s t}\right\}$ and the conservation variables $\{\boldsymbol{p}, \boldsymbol{F}, J\}$ are expanded in terms of nodal values and linear shape functions. It is possible to obtain the discrete set of nodal equations as

$$
\begin{aligned}
\sum_{b} \mathcal{M}_{a b} \dot{\boldsymbol{p}}_{b} & =\int_{\partial V} N_{a} \boldsymbol{t}^{B} d A+\int_{V} N_{a} \rho_{0} \boldsymbol{b} d V-\int_{V} \boldsymbol{P}^{s t} \nabla_{0} N_{a} d V, \\
\sum_{b} \mathcal{M}_{a b} \dot{\boldsymbol{F}}_{b} & =\int_{\partial V} N_{a}\left(\frac{\boldsymbol{p}^{B}}{\rho_{0}} \otimes \boldsymbol{N}\right) d A-\int_{V} \frac{\boldsymbol{p}_{\boldsymbol{F}}^{s t}}{\rho_{0}} \otimes \nabla_{0} N_{a} d V, \\
\sum_{b} \mathcal{M}_{a b} \dot{J}_{b} & =\int_{\partial V} \frac{\boldsymbol{p}^{B}}{\rho_{0}} \cdot \boldsymbol{H}_{\boldsymbol{F}} N_{a} \boldsymbol{N} d A-\int_{V} \frac{\boldsymbol{p}_{J}^{s t}}{\rho_{0}} \cdot \boldsymbol{H}_{\boldsymbol{F}} \nabla_{0} N_{a} d V .
\end{aligned}
$$

In equation (67a), $\boldsymbol{P}^{\text {st }}$ represents the stabilised first Piola-Kirchhoff stress tensor. This tensor, following a VMS approach [44], can be defined in terms of a stabilised deformation gradient $\boldsymbol{F}^{s t}$ and Jacobian $J^{s t}$ as

$$
\boldsymbol{P}^{s t}:=\boldsymbol{P}^{d e v}\left(\boldsymbol{F}^{s t}\right)+\kappa\left(J^{s t}-1\right) \boldsymbol{H}_{F},
$$

where

$$
\begin{aligned}
\boldsymbol{F}^{s t} & :=\boldsymbol{F}+\tau_{\boldsymbol{F} \boldsymbol{p}} \boldsymbol{\mathcal { R }}_{\boldsymbol{F}} \\
J^{s t} & :=J+\tau_{J \boldsymbol{p}} \boldsymbol{\mathcal { R }}_{J}
\end{aligned}
$$


Analogously, the stabilised linear momenta $\boldsymbol{p}_{\boldsymbol{F}}^{s t}$ and $\boldsymbol{p}_{J}^{s t}$ appearing in the discrete conservation equations $(67 \mathrm{~b}-67 \mathrm{c})$ are defined by

$$
\begin{aligned}
& \boldsymbol{p}_{\boldsymbol{F}}^{s t}=\boldsymbol{p}+\tau_{\boldsymbol{p} \boldsymbol{F}} \boldsymbol{\mathcal { R }}_{\boldsymbol{p}}, \\
& \boldsymbol{p}_{J}^{s t}=\boldsymbol{p}+\tau_{\boldsymbol{p} J} \boldsymbol{\mathcal { R }}_{\boldsymbol{p}} .
\end{aligned}
$$

For simplicity, the stabilisation factor $\tau_{\boldsymbol{F} J}$ has been taken as zero. Otherwise, the stabilised linear momentum $\boldsymbol{p}_{J}^{s t}$ would carry an extra contribution (refer to equation (64b)). In addition, for the speed up of the algorithm, the use of a lumped mass matrix is preferred. Moreover, in equation (67c), terms $\left(\frac{\boldsymbol{p}^{B}}{\rho_{0}} \cdot \boldsymbol{H}_{\boldsymbol{F}}\right)$ and $\left(\frac{\boldsymbol{p}_{J}^{s t}}{\rho_{0}} \cdot \boldsymbol{H}_{\boldsymbol{F}}\right)$ appearing in both integrands are linearly approximated within every finite element.

Traction and linear momentum at the boundary, $\boldsymbol{t}^{B}$ and $\boldsymbol{p}^{B}$, respectively, are evaluated from prescribed boundary conditions. As already described in section 2.3, the stabilisation parameter $\tau_{\boldsymbol{p} \boldsymbol{F}}$ is set to zero to ensure the discrete satisfaction of the involution of the deformation gradient tensor $\boldsymbol{F}$ (i.e. curl free condition).

Insofar as both the deformation gradient and the Jacobian are treated as independent unknowns in this mixed formulation, not explicitly related to the mapping $\boldsymbol{\phi}$, namely $\boldsymbol{F} \neq \boldsymbol{\nabla}_{0} \boldsymbol{x}$ and $J \neq \operatorname{det}\left(\boldsymbol{\nabla}_{0} \boldsymbol{x}\right)$, expressions (69a) and $(69 \mathrm{~b})$ can be further enhanced by adding the relevant time integrated stabilisations, namely $\int_{t} \tau_{\boldsymbol{F} \boldsymbol{p}} \boldsymbol{\mathcal { R }}_{\boldsymbol{F}} d t$ and $\int_{t} \tau_{J \boldsymbol{p}} \boldsymbol{\mathcal { R }}_{J} d t$, to penalise the difference between $\boldsymbol{F}$ and $\boldsymbol{\nabla}_{0} \boldsymbol{x}$ and the difference between $J$ and $\operatorname{det}\left(\boldsymbol{\nabla}_{0} \boldsymbol{x}\right)$, to yield

$$
\begin{aligned}
\boldsymbol{F}^{s t} & =\boldsymbol{F}+\tau_{\boldsymbol{F} \boldsymbol{p}} \boldsymbol{\mathcal { R }}_{\boldsymbol{F}}+\alpha\left(\boldsymbol{\nabla}_{0} \boldsymbol{x}-\boldsymbol{F}\right), \\
J^{s t} & =J+\tau_{J \boldsymbol{p}} \frac{\mu}{\kappa} \boldsymbol{\mathcal { R }}_{J}+\beta \frac{\mu}{\kappa}\left(\operatorname{det}\left(\boldsymbol{\nabla}_{0} \boldsymbol{x}\right)-J\right),
\end{aligned}
$$

where $\alpha$ and $\beta$ are non-dimensional stabilisation parameters in the range 0 to 0.5 . The dimensionless ratio $\frac{\mu}{\kappa}$ present in equation (71b) is introduced for consistency with the fractional step approach to be presented in a subsequent section. The residual based $\alpha$ - and $\beta$-terms present in (71a-71b) provide additional stability and, more importantly, help reducing the level of implicitness of the formulation.

Notice that when $\tau_{J p}=0$, equations $(67 \mathrm{a}-67 \mathrm{c})$ are fully decoupled and can be solved in a sequential manner. Equation $(67 \mathrm{~b})$ is first solved to obtain $\dot{\boldsymbol{F}}$ which can then be substituted into (67a) to deduce $\dot{\boldsymbol{p}}$. Once $\dot{\boldsymbol{p}}$ is determined, $\dot{J}$ can finally be obtained from (67c). Unlike a two-step Taylor Galerkin formulation [45], all weighted residual based stabilising parameters can be 
suitably selected in an independent manner enhancing the robustness of the scheme.

The stabilised semidiscrete nodal equations (67a-67c) are then explicitly integrated from time step $t^{n}$ to $t^{n+1}$ with the TVD-RK time integrator [72]. For stability, the maximum time increment $\Delta t$ is evaluated based on the minimum size of element $h_{\min }$ and the maximum wave speed $c_{\max }=c_{p}(60)$.

\subsection{Fractional step approach: large deformation}

The explicit $\boldsymbol{p}-\boldsymbol{F}$ - $J$ Petrov-Galerkin (PG) formulation presented above is not computationally suitable to model nearly (fully) incompressible materials as the volumetric wave speed can be significantly high (infinity) leading to a time step size $\Delta t$ extremely small (zero). In these situations, it is preferred to resolve the incompressibility constraint in an implicit manner. The maximum time step size is then limited by the shear wave speed $c_{s}$.

The conservation equation for the Jacobian (41c) is replaced by the constraint $\boldsymbol{H}_{\boldsymbol{F}}: \boldsymbol{\nabla}_{0}\left(\frac{\boldsymbol{p}}{\rho}\right)=0$. In this case, the new unknowns for the problem are $\{\boldsymbol{p}, \boldsymbol{F}, p\}$ ( $J$ is replaced with $p)$, as the Jacobian is constrained to be always one throughout the entire deformation process. A predictor-corrector algorithm is designed to advance the problem unknowns from $t^{n}$ to $t^{n+1}$ in such a way that only the pressure field $p$ is solved implicitly in time $t^{n+1}$. The predictor step is of the form

$$
\begin{aligned}
\frac{\left(\boldsymbol{p}^{i n t}-\boldsymbol{p}^{n}\right)}{\Delta t}-\operatorname{DIV} \boldsymbol{P}^{d e v, n}-\operatorname{DIV}\left(p^{n} \boldsymbol{H}_{\boldsymbol{F}}{ }^{n}\right)-\rho_{0} \boldsymbol{b}^{n} & =\mathbf{0}, \\
\frac{\boldsymbol{F}^{n+1}-\boldsymbol{F}^{n}}{\Delta t}-\nabla_{0}\left(\frac{\boldsymbol{p}^{n}}{\rho_{0}}\right) & =\mathbf{0},
\end{aligned}
$$

and the corrector step

$$
\frac{\left(\boldsymbol{p}^{n+1}-\boldsymbol{p}^{i n t}\right)}{\Delta t}-\mathrm{DIV}\left[\left(p^{n+1}-p^{n}\right) \boldsymbol{H}_{\boldsymbol{F}}^{n}\right]=\mathbf{0} .
$$

Notice that the summation of (72a) and (73) recovers the original assumption that only the pressure field $p$ is computed implicitly in $t^{n+1}$ (i.e. the cofactor $\boldsymbol{H}_{\boldsymbol{F}}$ is frozen at time $\left.t^{n}\right)$. Application of the operator $\boldsymbol{H}_{\boldsymbol{F}}{ }^{n}: \boldsymbol{\nabla}_{0}(\cdot)$ to above equation (73) yields,

$\boldsymbol{H}_{\boldsymbol{F}}{ }^{n}: \boldsymbol{\nabla}_{0}\left(\frac{\boldsymbol{p}^{n+1}}{\rho_{0}}\right)-\boldsymbol{H}_{\boldsymbol{F}}{ }^{n}: \boldsymbol{\nabla}_{0}\left(\frac{\boldsymbol{p}^{i n t}}{\rho_{0}}\right)-\frac{\Delta t}{\rho_{0}} \boldsymbol{H}_{\boldsymbol{F}}{ }^{n}: \nabla_{0}\left[\mathrm{DIV}\left(\left(p^{n+1}-p^{n}\right) \boldsymbol{H}_{\boldsymbol{F}}{ }^{n}\right)\right]=0$. 
For simplicity and without loss of generality, the material density $\rho_{0}$ has been assumed to be constant across the entire domain. To allow for the case of a nearly incompressible NH material, we can write

$$
\boldsymbol{H}_{\boldsymbol{F}}{ }^{n}: \boldsymbol{\nabla}_{0}\left(\frac{\boldsymbol{p}^{n+1}}{\rho_{0}}\right)=\frac{p^{n+1}-p^{n}}{\kappa \Delta t} .
$$

For a truly incompressible material, $\kappa=\infty$ and the right hand side of above equation vanishes resulting in the incompressibility constraint. Using this relationship on the first term of (74), it yields

$$
\frac{p^{n+1}-p^{n}}{\kappa \Delta t}-\boldsymbol{H}_{\boldsymbol{F}}{ }^{n}: \boldsymbol{\nabla}_{0}\left(\frac{\boldsymbol{p}^{i n t}}{\rho_{0}}\right)-\frac{\Delta t}{\rho_{0}} \boldsymbol{H}_{\boldsymbol{F}}{ }^{n}: \boldsymbol{\nabla}_{0}\left[\mathrm{DIV}\left(\left(p^{n+1}-p^{n}\right) \boldsymbol{H}_{\boldsymbol{F}}{ }^{n}\right)\right]=0 \text {. }
$$

\subsubsection{Variational nonlinear fractional step formulation}

To obtain a variational statement, we first define the residuals of equations (72a), (72b) and (76) as

$$
\begin{aligned}
\mathcal{R}_{\boldsymbol{p}^{i n t}} & :=\operatorname{DIV} \boldsymbol{P}^{d e v, n}+\operatorname{DIV}\left(p^{n} \boldsymbol{H}_{\boldsymbol{F}}{ }^{n}\right)+\rho_{0} \boldsymbol{b}^{n}-\frac{\left(\boldsymbol{p}^{i n t}-\boldsymbol{p}^{n}\right)}{\Delta t}, \\
\mathcal{R}_{\boldsymbol{F}} & :=\boldsymbol{\nabla}_{0}\left(\frac{\boldsymbol{p}^{n}}{\rho_{0}}\right)-\frac{\boldsymbol{F}^{n+1}-\boldsymbol{F}^{n}}{\Delta t}, \\
\mathcal{R}_{J} & :=\boldsymbol{H}_{\boldsymbol{F}}{ }^{n}: \boldsymbol{\nabla}_{0}\left(\frac{\boldsymbol{p}^{i n t}}{\rho_{0}}\right)+\frac{\Delta t}{\rho_{0}} \boldsymbol{H}_{\boldsymbol{F}}^{n}: \boldsymbol{\nabla}_{0}\left[\mathrm{DIV}\left(\left(p^{n+1}-p^{n}\right) \boldsymbol{H}_{\boldsymbol{F}}^{n}\right)\right]-\frac{p^{n+1}-p^{n}}{\kappa \Delta t} \\
\boldsymbol{\mathcal { R }}_{\boldsymbol{p}} & :=\operatorname{DIV}\left(\left(p^{n+1}-p^{n}\right) \boldsymbol{H}_{\boldsymbol{F}}^{n}\right)-\frac{\left(\boldsymbol{p}^{n+1}-\boldsymbol{p}^{i n t}\right)}{\Delta t}
\end{aligned}
$$

Using appropriate stabilised conjugate virtual fields $\mathcal{V}^{s t}=\left[\delta \boldsymbol{v}^{s t}, \delta \boldsymbol{P}^{s t}, \delta q^{s t}\right]^{T}$, already defined in (64), a variational statement is defined by

$$
\delta W^{\mathrm{PG}}=\int_{V} \delta \boldsymbol{v}^{s t} \cdot \boldsymbol{\mathcal { R }}_{\boldsymbol{v}^{\text {int }}} d V+\int_{V} \delta \boldsymbol{P}^{s t}: \boldsymbol{\mathcal { R }}_{\boldsymbol{F}} d V+\int_{V} \delta q^{s t} \mathcal{R}_{J} d V=0,
$$

Following a similar finite element spatial discretisation as that presented in the previous section, where $\{\boldsymbol{p}, \boldsymbol{F}, p\}$ and $\left\{\delta \boldsymbol{v}^{s t}, \delta \boldsymbol{P}^{s t}, \delta q^{s t}\right\}$ are expanded in terms of linear shape functions, the resulting predictor system of equations 
yields

$$
\begin{aligned}
\sum_{b} \mathcal{M}_{a b} \frac{\left(\boldsymbol{p}_{b}^{i n t}-\boldsymbol{p}_{b}^{n}\right)}{\Delta t} & =\int_{\partial V} N_{a} \boldsymbol{t}^{B, n} d A+\int_{V} N_{a} \rho_{0} \boldsymbol{b}^{n} d V-\int_{V} \boldsymbol{P}\left(\boldsymbol{F}^{s t}, p^{s t}\right) \boldsymbol{\nabla}_{0} N_{a} d V \\
\sum_{b} \mathcal{M}_{a b} \frac{\left(\boldsymbol{F}_{b}^{n+1}-\boldsymbol{F}_{b}^{n}\right)}{\Delta t} & =\int_{\partial V} N_{a}\left(\frac{\boldsymbol{p}^{B, n}}{\rho_{0}} \otimes \boldsymbol{N}\right) d A-\int_{V} \frac{\boldsymbol{p}_{\boldsymbol{F}}^{s t}}{\rho_{0}} \otimes \nabla_{0} N_{a} d V
\end{aligned}
$$

where

$$
\begin{aligned}
\boldsymbol{F}^{s t} & :=\boldsymbol{F}^{n}+\tau_{\boldsymbol{F} \boldsymbol{p}} \mathcal{R}_{\boldsymbol{F}}+\alpha\left(\boldsymbol{\nabla}_{0} \boldsymbol{x}^{n}-\boldsymbol{F}^{n}\right), \\
p^{s t} & :=p^{n}+\tau_{J \boldsymbol{p}} \mu \mathcal{R}_{J}+\beta \mu\left(\operatorname{det}\left(\boldsymbol{\nabla}_{0} \boldsymbol{x}^{n}\right)-1-\frac{p^{n}}{\kappa}\right), \\
\boldsymbol{p}_{\boldsymbol{F}}^{s t} & :=\boldsymbol{p}^{n}+\tau_{\boldsymbol{p} \boldsymbol{F}} \boldsymbol{\mathcal { R }}_{\boldsymbol{p}}^{i n t} .
\end{aligned}
$$

Analogously to section 2.4.1, by using linear shape functions for the expansion of $\left(p^{n+1}-p^{n}\right) \boldsymbol{H}_{\boldsymbol{F}}{ }^{n}$ in the second term of the right hand side of equation (77c), this term vanishes. For an incompressible medium where $\kappa=\infty$ the term in parenthesis on the right hand side of $(80 \mathrm{~b})$ reduces to the volumetric constraint. This equation (80b) is consistent with $(71 \mathrm{~b})$, as multiplication of (71b) by $\kappa$ yields $(80 \mathrm{~b})$.

Setting $\tau_{\boldsymbol{p} \boldsymbol{F}}=\tau_{J \boldsymbol{p}}=0$, equations (79a-79b) can be solved sequentially. The corrector step is formulated as

$$
\begin{aligned}
\sum_{b}\left[\mathcal{M}_{a b}^{v o l}+\frac{\Delta t^{2}}{\rho_{0}} \mathcal{K}_{a b}\right]\left(\frac{p_{b}^{n+1}-p_{b}^{n}}{\Delta t}\right) d V & =\int_{\partial V}\left(\frac{\boldsymbol{p}^{B}}{\rho_{0}} \cdot \boldsymbol{H}_{\boldsymbol{F}}{ }^{n}\right) N_{a} \boldsymbol{N} d A \\
& -\int_{V}\left(\frac{\boldsymbol{p}_{J}^{s t}}{\rho_{0}} \cdot \boldsymbol{H}_{\boldsymbol{F}}{ }^{n}\right) \nabla_{0} N_{a} d V
\end{aligned}
$$

where the mas matrix contribution $\mathcal{M}_{a b}^{v o l}$, the viscosity matrix contribution $\mathcal{K}_{a b}$ and the stabilised linear momentum $\boldsymbol{p}_{J}^{s t}$ are defined as

$$
\begin{aligned}
\mathcal{M}_{a b}^{\text {vol }} & :=\int_{V} \frac{1}{\kappa} N_{a} N_{b} d V \\
\mathcal{K}_{a b} & :=\int_{V}\left(\boldsymbol{H}_{\boldsymbol{F}}{ }^{n} \boldsymbol{\nabla}_{0} N_{a}\right) \cdot\left(\boldsymbol{H}_{\boldsymbol{F}}{ }^{n} \boldsymbol{\nabla}_{0} N_{b}\right) d V \\
\boldsymbol{p}_{J}^{s t} & :=\boldsymbol{p}^{i n t}+\tau_{\boldsymbol{p} J} \boldsymbol{\mathcal { R }}_{\boldsymbol{p}^{i n t}},
\end{aligned}
$$


respectively. In addition, in equation (81), terms $\left(\frac{\boldsymbol{p}^{B}}{\rho_{0}} \cdot \boldsymbol{H}_{\boldsymbol{F}}{ }^{n}\right)$ and $\left(\frac{\boldsymbol{p}_{J}^{s t}}{\rho_{0}} \cdot \boldsymbol{H}_{\boldsymbol{F}}{ }^{n}\right)$ appearing in both integrands in the right hand side are linearly approximated within every finite element. It is now possible to update the linear momentum $\boldsymbol{p}^{n+1}$ using the conventional Bubnov-Galerkin (BG) formulation $\delta W^{\mathrm{BG}}=\int_{V} \delta \boldsymbol{v} \cdot \boldsymbol{\mathcal { R }}_{\boldsymbol{p}} d V$ to give

$$
\sum_{b} \mathcal{M}_{a b} \frac{\boldsymbol{p}_{b}^{n+1}-\boldsymbol{p}_{b}^{i n t}}{\Delta t}=\int_{V} N_{a} \operatorname{DIV}\left(\left(p^{n+1}-p^{n}\right) \boldsymbol{H}_{\boldsymbol{F}}{ }^{n}\right) d V
$$

The algorithm is finally evolved in time via a TVD-RK time integrator with a time step limit controlled by the shear wave speed $c_{s}$

\section{Numerical examples}

In this section, a number of numerical examples will be presented in order to assess the performance of the enhanced $\boldsymbol{p}-\boldsymbol{F}-J(\boldsymbol{p}-\boldsymbol{G}-j$ in small deformations) Petrov-Galerkin (PG) formulation in compressible, nearly incompressible and truly incompressible regimes, compared against the stabilised $\boldsymbol{p}-\boldsymbol{F}$ $(\boldsymbol{p}-\boldsymbol{G})$ formulation [44], together with the use of an explicit two stage Total Variation Diminishing Runge-Kutta (TVD-RK) time integrator.

\subsection{Low dispersion cube}

This example is an extension of the two dimensional plate presented in [44] to assess the convergence behaviour of the algorithm in three dimensions [71]. A unit cube $(1 \mathrm{~m} \times 1 \mathrm{~m} \times 1 \mathrm{~m})$ has symmetric boundary conditions (i.e. roller supports) applied on the faces $x_{1}=x_{2}=x_{3}=0 \mathrm{~m}$ and skew-symmetric boundary conditions on the rest of the boundary surfaces $x_{1}=x_{2}=x_{3}=1 \mathrm{~m}$. For small deformations, the analytical displacement field (and hence velocity and Cauchy stress at any time $t$ ) can be described by a closed-form expression as

$$
\boldsymbol{u}(\boldsymbol{x}, t)=U_{0} \cos \left(\frac{\sqrt{3}}{2} c_{d} \pi t\right)\left[\begin{array}{c}
A \sin \left(\frac{\pi x_{1}}{2}\right) \cos \left(\frac{\pi x_{2}}{2}\right) \cos \left(\frac{\pi x_{3}}{2}\right) \\
B \cos \left(\frac{\pi x_{1}}{2}\right) \sin \left(\frac{\pi x_{2}}{2}\right) \cos \left(\frac{\pi x_{3}}{2}\right) \\
C \cos \left(\frac{\pi x_{1}}{2}\right) \cos \left(\frac{\pi x_{2}}{2}\right) \sin \left(\frac{\pi x_{3}}{2}\right)
\end{array}\right] ; c_{d}=\sqrt{\frac{\mu}{\rho_{0}}}
$$


Parameters $\{A, B, C\}$ are arbitrary constants such that $A+B+C=0$, ensuring no contribution from volumetric deformation. For values of $U_{0}$ below 0.001, the solution can be considered to be linear and the closedform solution holds. The cube is initially loaded with a known displacement gradient field $G^{0}$ (by computing the gradient of (84) at $t=0$ ) without any initial velocity. In this particular case, a linear constitutive model is chosen such that Young's modulus $E=0.017 \mathrm{GPa}$, density $\rho_{0}=1.1 \mathrm{Mg} / \mathrm{m}^{3}$ and Poisson's ratio $\nu=0.45$. We set the solution parameters as $A=B=1$, $C=-2$ and $U_{0}=5 \times 10^{-4} \mathrm{~m}$ in linear regime. Global convergence error analysis for velocity and stress on a sequence of grids at time $t=2 \times 10^{-3}$ s are examined in Figure 1. As expected, each of the diagrams tends to asymptotic quadratic convergence for all the variables of the $\boldsymbol{p}$ - $\boldsymbol{G}$ - $\boldsymbol{j}$ formulation when linear interpolating functions are used.

\subsection{Beam bending}

The small bending deformation of a cantilever beam with dimensions $1 \times 1 \times 10 \mathrm{~m}$ is studied. The beam is fully clamped on one end and loaded with a sudden constant tip traction of $P_{0}=-1 \times 10^{-5} \mathrm{~Pa}$ on the other end. A linear elastic constitutive model in which Young's modulus $E=1 \mathrm{~Pa}$ and density $\rho_{0}=0.1 \mathrm{~kg} / \mathrm{m}^{3}$ is chosen. The purpose of this example is to illustrate the performance of the proposed methodologies in bending dominated scenarios in linear compressible regimes by imposing Poisson's ratio $\nu=0.3$. In these circumstances, the recently proposed $\boldsymbol{p}$ - $\boldsymbol{G}$ formulation [44] produces reliable results, which can be treated as reference solutions for comparison purposes. Figure 2 shows the position of a cantilever beam at various time instants for three cases: standard $\boldsymbol{p}-\boldsymbol{G}$ PG formulation, linearised $\boldsymbol{p}-\boldsymbol{G}-\boldsymbol{j}$ PG formulation and linearised $\boldsymbol{p}-\boldsymbol{G}-\boldsymbol{p}$ fractional step PG formulation. It is clear that the proposed algorithms can be used without bending difficulty. More importantly, the deformed shapes obtained from the enhanced methodologies are found to be in perfect agreement with the standard $\boldsymbol{v}-\boldsymbol{G}$ approach.

\subsection{Thick column}

A three dimensional thick column [71] clamped on its bottom face $\left(X_{3}=\right.$ 0 ) is presented in this numerical example (see Figure 3). An initial linear variation in velocity field $\boldsymbol{v}^{0}=\left(V_{0} X_{3} / L, 0,0\right)^{T}$ is given (where $V_{0}=10 \mathrm{~m} / \mathrm{s}$ ) and the column is left oscillating in time, leading to large strain oscillatory motion. The objective is to assess the performance of the different large deformation PG methodologies (i.e. $\boldsymbol{p}$ - $\boldsymbol{F}$ formulation, $\boldsymbol{p}$ - $\boldsymbol{F}$ - $J$ formulation 

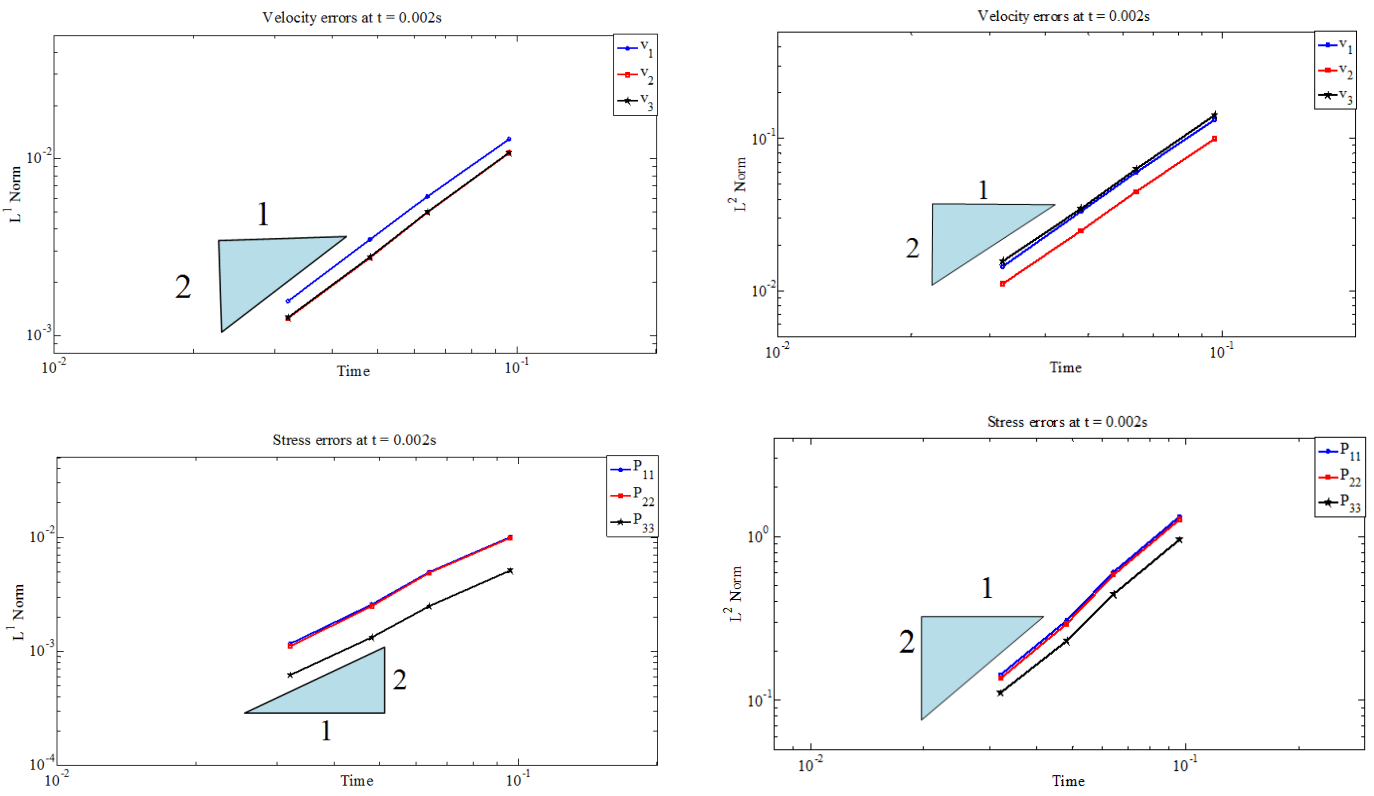

Figure 1: Low Dispersion Cube: Results obtained with $U_{0}=5 \times 10^{-4} \mathrm{~m}$ where the analytical solutions for velocity and stress are available at time $t=$ $2 \times 10^{-3}$ s. First column shows the $\mathrm{L}^{1}$ norm convergence and second column shows the $\mathrm{L}^{2}$ norm convergence. First row shows the velocity errors and second row illustrates the stress errors. The linear elastic model is used and material properties are such that Young's modulus $E=0.017 \mathrm{GPa}$, density $\rho_{0}=1.1 \mathrm{Mg} / \mathrm{m}^{3}$, Poisson's ratio $\nu=0.45$ and $\alpha_{C F L}=0.4$. Stabilising parameters of the $\boldsymbol{p}-\boldsymbol{G}-j \mathrm{PG}$ formulation using lumped mass matrix are: $\tau_{\boldsymbol{G} \boldsymbol{v}}=\Delta t, \tau_{\boldsymbol{v} j}=0.1 \Delta t, \alpha=0$ and $\beta=0.5$. 

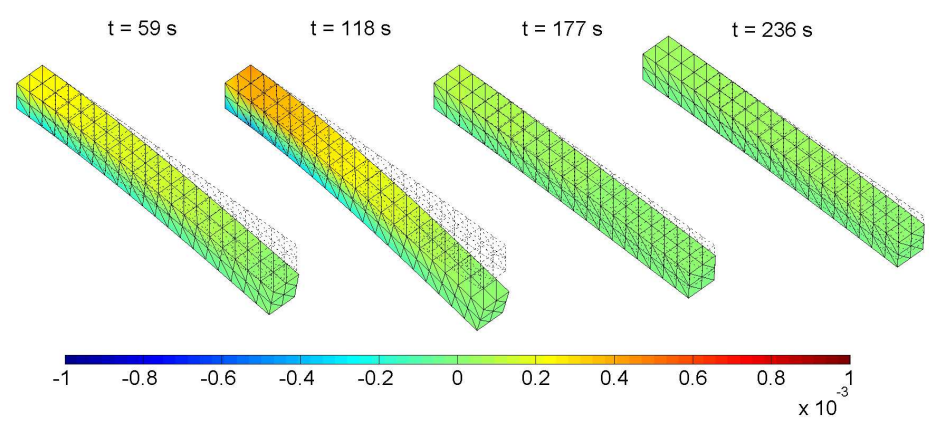

(a) Standard $\boldsymbol{p}-\boldsymbol{G}\left(\tau_{\boldsymbol{F}}=\Delta t, \tau_{\boldsymbol{p}}=0, \alpha=0.1\right)$
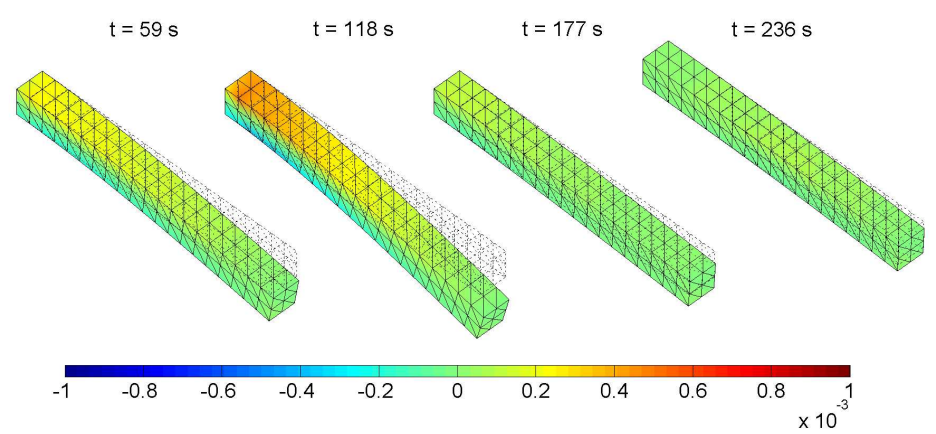

(b) Linearised $\boldsymbol{p}$ - $\boldsymbol{G}-j\left(\tau_{\boldsymbol{G} \boldsymbol{p}}=\Delta t, \tau_{\boldsymbol{p} j}=0.2 \Delta t, \alpha=0, \beta=0.5\right)$

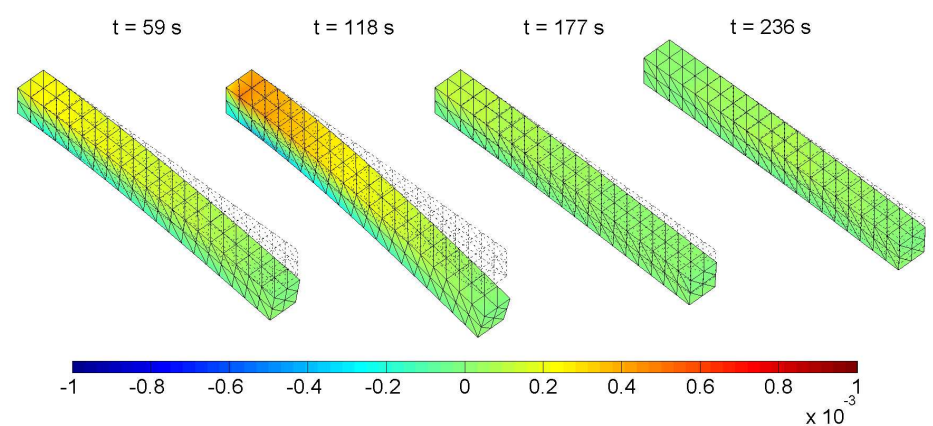

(c) Linear fractional step $\left(\tau_{\boldsymbol{G} \boldsymbol{p}}=\Delta t, \tau_{\boldsymbol{p} j}=0.2 \Delta t, \alpha=0, \beta=0.5\right)$

Figure 2: Cantilever Beam: Sequence of deformed shapes (pressure contour plot) using (a) Standard $\boldsymbol{p}$ - $\boldsymbol{G}$ PG formulation; (b) Linearised $\boldsymbol{p}$ - $\boldsymbol{G}$ - $\boldsymbol{j}$ PG formulation; and (c) Linearised fractiBonal step PG formulation. Results obtained with a sudden constant tip traction $P_{0}=1 \times 10^{-5} \mathrm{~Pa}$. A linearised constitutive model is used such that Young's modulus $E=1 \mathrm{~Pa}$, density $\rho_{0}=0.1 \mathrm{~kg} / \mathrm{m}^{3}$, Poisson's ratio $\nu=0.3$ and $\alpha_{C F L}=0.3$. Discretisation of $2 \times 2 \times 20 \times 6$ tetrahedral elements. Lumped mass contribution. Solution plotted with displacement scaled 20 times. 
and fractional step formulation) in bending difficulty. A Neo-Hookean (NH) material model is employed such that Young's modulus $E=0.017 \mathrm{GPa}$ and density $\rho_{0}=1.1 \mathrm{Mg} / \mathrm{m}^{3}$. We examine the proposed schemes in compressible regime by taking the Poisson's ratio $\nu=0.45$.

Both the enhanced $\boldsymbol{p}-\boldsymbol{F}-J$ and the fractional step PG formulations show excellent agreement with the existing $\boldsymbol{p}$ - $\boldsymbol{F}$ formulation (see Figure 4 and Figure 5). For truly incompressible solids, the explicit $\boldsymbol{p}-\boldsymbol{F}$ and $\boldsymbol{p}-\boldsymbol{F}-J$ formulations cannot be used due to the incompressibility constraint $(\kappa=\infty)$. Such limitation is removed by employing the fractional step PG formulation in which the time step depends only on the shear wave speed (see Figure 6). In all cases, the pressure contours are extremely smooth and without spurious oscillations.

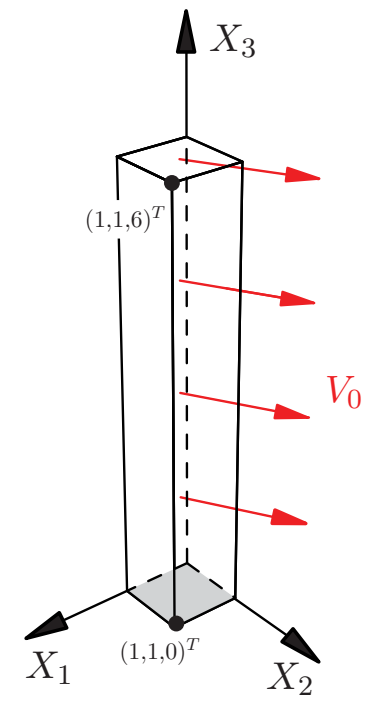

Figure 3: Thick column configuration

\subsection{Twisting column}

Similar to the case of bending column discussed in Section 4.3, a twisting column clamped on its bottom face is presented (see Figure 7). An initial sinusoidal rotational velocity field relative to the origin is given by

$$
\boldsymbol{v}^{0}(\boldsymbol{X})=\boldsymbol{\omega} \times \boldsymbol{X} ; \quad \boldsymbol{\omega}=\left(0,0, \Omega \sin \left(\frac{\Pi X_{3}}{2 L}\right)\right)^{T}
$$




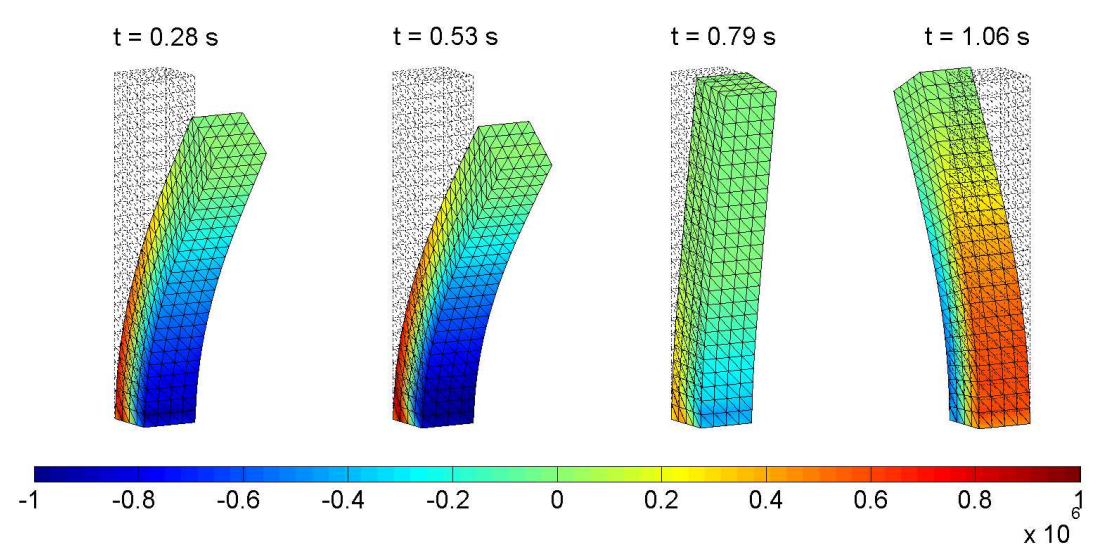

(a) $\boldsymbol{p}$ - $\boldsymbol{F}$-J PG formulation

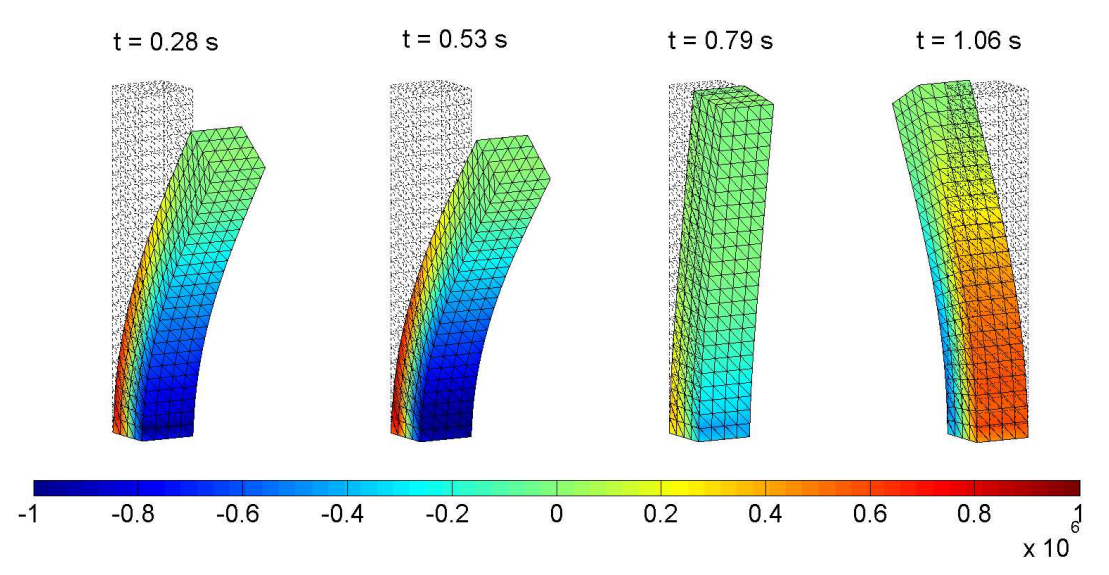

(b) Fractional step PG formulation

Figure 4: Bending Column: Sequence of deformed shapes (pressure contour plot) using (a) $\boldsymbol{p}-\boldsymbol{F}-J$ PG formulation; and (b) Nonlinear fractional step $\mathrm{PG}$ formulation. Results obtained with a linear variation in velocity field $\boldsymbol{v}^{0}=\left(V_{0} X_{3} / L, 0,0\right)^{T}$ where $V_{0}=10 \mathrm{~m} / \mathrm{s}$. The nearly incompressible NeoHookean (NH) constitutive model is used such that Young's modulus $E=$ $0.017 \mathrm{GPa}$, density $\rho_{0}=1.1 \mathrm{Mg} / \mathrm{m}^{3}$, Poisson's ratio $\nu=0.45$ and $\alpha_{C F L}=0.3$. Discretisation of $4 \times 4 \times 24 \times 6$ tetrahedral elements. Stabilising parameters: $\tau_{\boldsymbol{F p}}=\Delta t, \tau_{\boldsymbol{p} J}=0.2 \Delta t, \alpha=0$ and $\beta$ z20.5. Lumped mass contribution. 


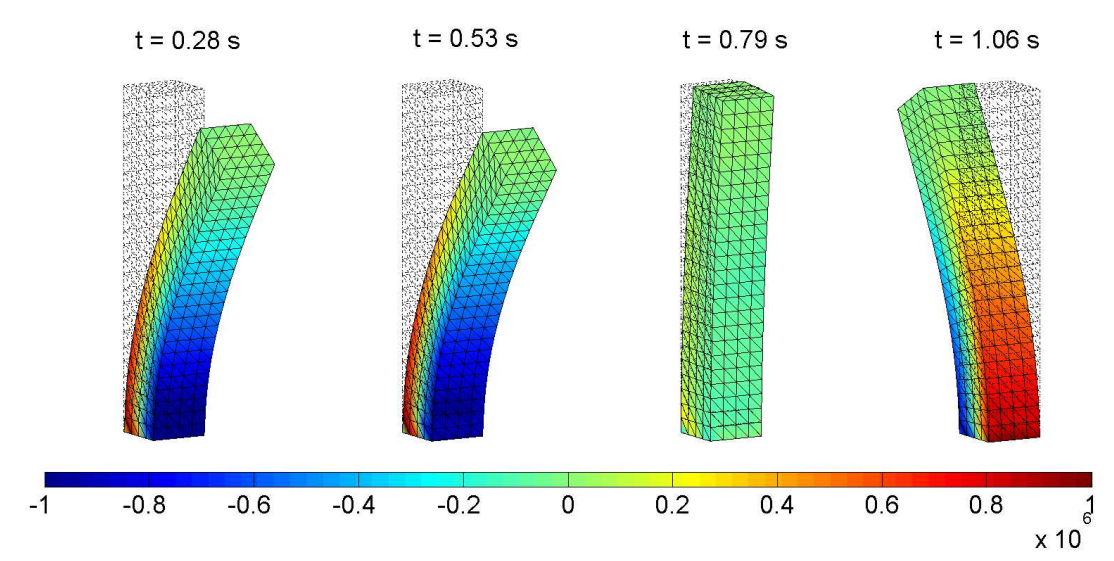

Figure 5: Bending Column: Sequence of deformed shapes (pressure contour plot) using $\boldsymbol{p}-\boldsymbol{F}$ PG formulation. Results obtained with a linear variation in velocity field $\boldsymbol{v}^{0}=\left(V_{0} X_{3} / L, 0,0\right)^{T}$ where $V_{0}=10 \mathrm{~m} / \mathrm{s}$. The nearly incompressible Neo-Hookean $(\mathrm{NH})$ constitutive model is used such that Young's modulus $E=0.017 \mathrm{GPa}$, density $\rho_{0}=1.1 \mathrm{Mg} / \mathrm{m}^{3}$, Poisson's ratio $\nu=0.45$ and $\alpha_{C F L}=0.3$. Discretisation of $4 \times 4 \times 24 \times 6$ tetrahedral elements. Stabilising parameters: $\tau_{\boldsymbol{F}}=\Delta t, \tau_{\boldsymbol{p}}=0$ and $\alpha=0.1$. Lumped mass contribution. 

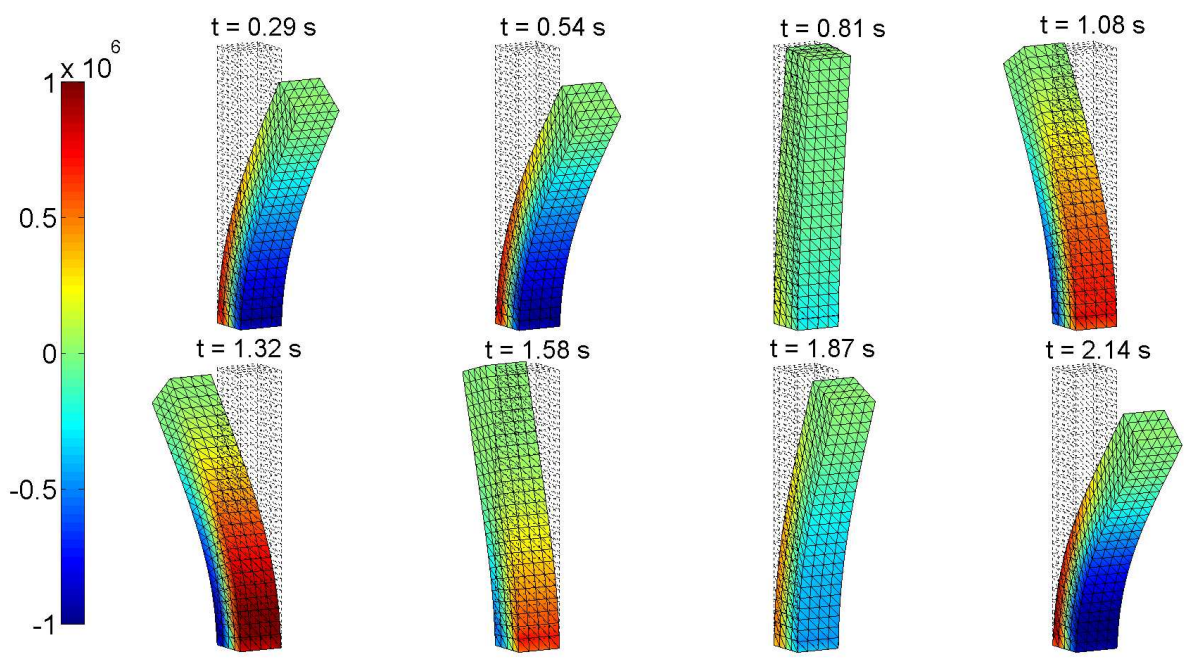

Figure 6: Bending Column: Sequence of deformed shapes (pressure contour plot) using nonlinear fractional step PG formulation. Results obtained with a linear variation in velocity field $\boldsymbol{v}^{0}=\left(V_{0} X_{3} / L, 0,0\right)^{T}$ where $V_{0}=10 \mathrm{~m} / \mathrm{s}$. Incompressible Neo-Hookean (NH) constitutive model is used such that Young's modulus $E=0.017 \mathrm{GPa}$, density $\rho_{0}=1.1 \mathrm{Mg} / \mathrm{m}^{3}$, Poisson's ratio $\nu=0.5$ and $\alpha_{C F L}=0.3$. Discretisation of $4 \times 4 \times 24 \times 6$ tetrahedral elements. Stabilising parameters: $\tau_{\boldsymbol{F} \boldsymbol{p}}=\Delta t, \tau_{\boldsymbol{p} J}=0.2 \Delta t, \alpha=0$ and $\beta=0.5$. Lumped mass contribution. 
where $\Omega=100 \mathrm{rad} / \mathrm{s}$. The main objective is to assess the robustness of the proposed algorithms in extremely high nonlinear deformations. This problem is modelled by using a nearly incompressible Neo-Hookean (NH) constitutive model where Young's modulus $E=0.017 \mathrm{GPa}$, material density $\rho_{0}=1.1 \mathrm{Mg} / \mathrm{m}^{3}$ and Poisson's ratio $\nu=0.499$. For comparison purposes, the performance of three different numerical strategies, namely $\boldsymbol{p}-\boldsymbol{F}, \boldsymbol{p}-\boldsymbol{F}-J$ and nonlinear fractional step PG formulations, are presented.

Clearly, the existing $\boldsymbol{p}-\boldsymbol{F}$ formulation exhibits non-physical pressure fluctuations due to insufficient numerical stabilisation and then follows a completely wrong solution path (see Figure 8). On the contrary, the use of the $\boldsymbol{p}-\boldsymbol{F}-J$ formulation and that of the fractional step implementation produce practically identical locking-free solutions that are freed from low-energy modes (see Figure 9). As can be observed, the pressure contours are very smooth. As expected, the fractional step solution is slightly more dissipative due to the contribution of the viscosity matrix.

We can further examine the performance of the fractional step PG formulation in the case of a truly incompressible material. Figure 10 illustrates the evolution of deformed shapes using the fractional step method, demonstrating the robustness of the proposed methodology.

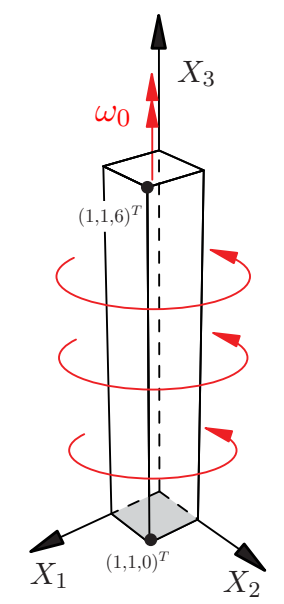

Figure 7: Highly nonlinear twisting column 


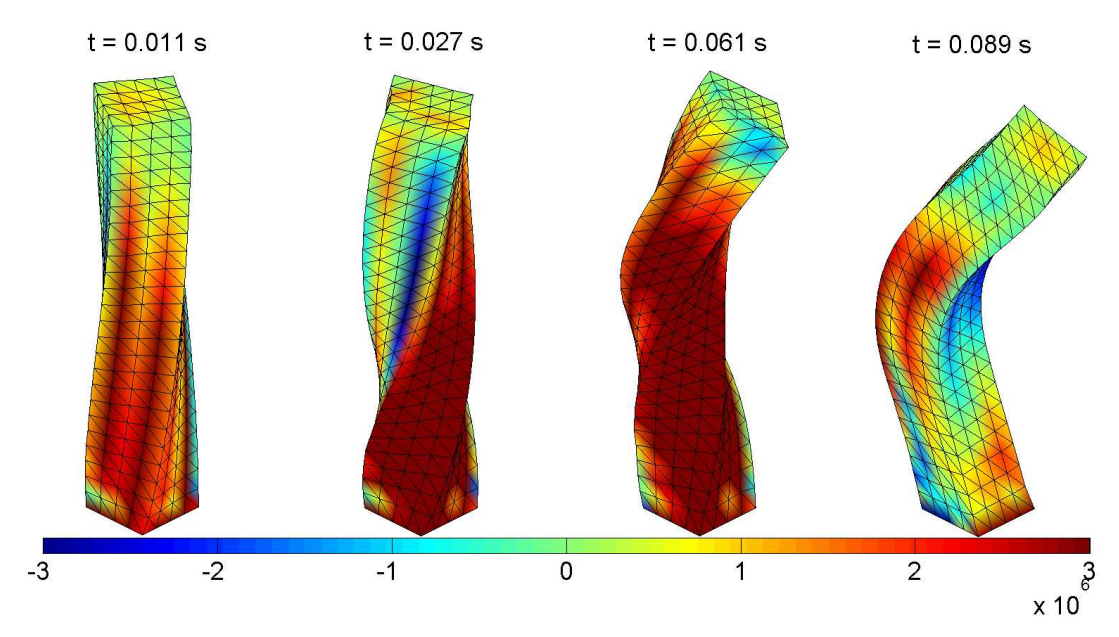

Figure 8: Twisting Column: Sequence of deformed shapes (pressure contour plot) using standard $\boldsymbol{p}-\boldsymbol{F}$ PG formulation. Results obtained with a initial sinusoidal rotational velocity $\Omega=100 \mathrm{rad} / \mathrm{s}$. A nearly incompressible NeoHookean $(\mathrm{NH})$ constitutive model is used such that Young's modulus $E=$ $0.017 \mathrm{GPa}$, density $\rho_{0}=1.1 \mathrm{Mg} / \mathrm{m}^{3}$, Poisson's ratio $\nu=0.499$ and $\alpha_{C F L}=0.3$. Stabilising parameters: $\tau_{\boldsymbol{F}}=\Delta t, \tau_{\boldsymbol{p}}=0.2 \Delta t$ and $\alpha=0.1$. Lumped mass contribution. 


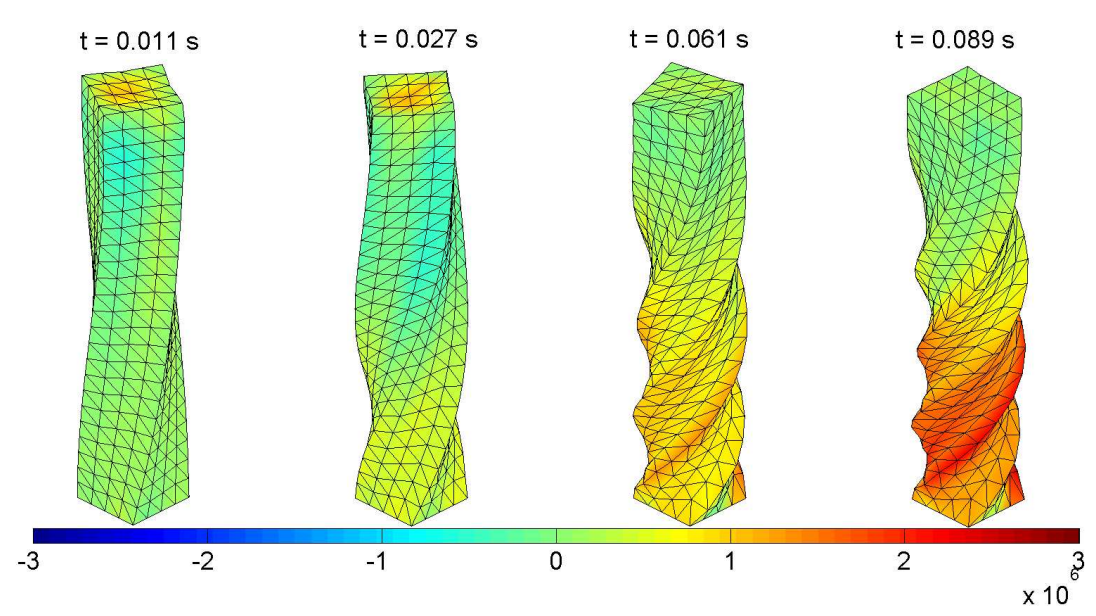

(a) Nearly incompressible $\boldsymbol{p}-\boldsymbol{F}-J$ formulation

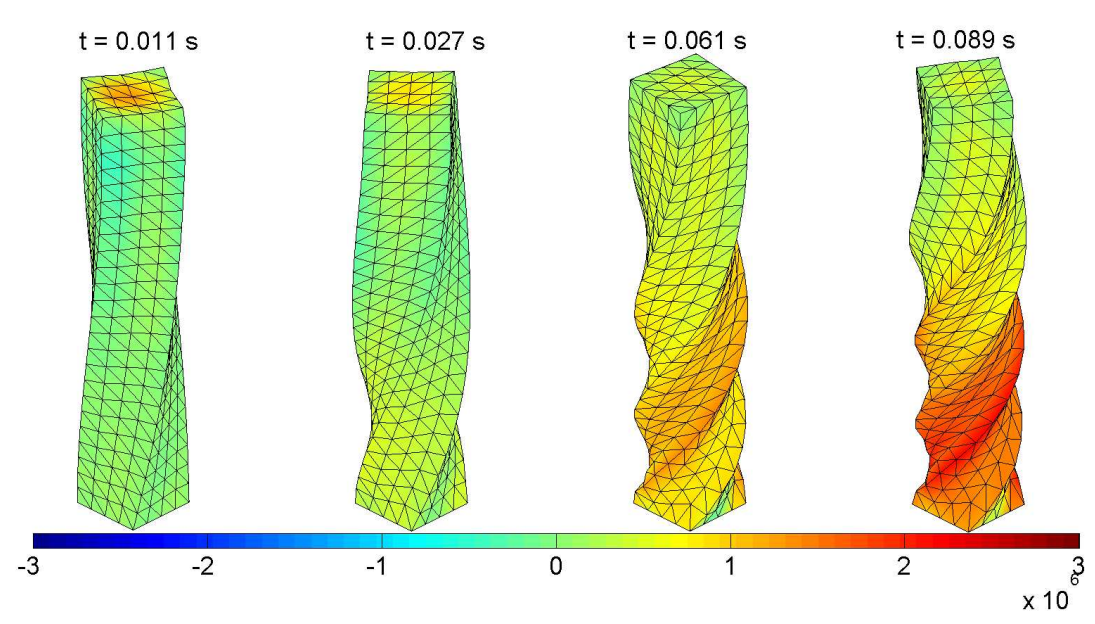

(b) Nearly incompressible fractional step formulation

Figure 9: Twisting Column: Sequence of deformed shapes (pressure contour plot) using (a) Nearly incompressible $\boldsymbol{p}-\boldsymbol{F}-J$ formulation; and (b) Nonlinear fractional step formulation. Results obtained with a initial sinusoidal rotational velocity $\Omega=100 \mathrm{rad} / \mathrm{s}$. A nearly incompressible Neo-Hookean (NH) constitutive model is used such that Young's modulus $E=0.017 \mathrm{GPa}$, density $\rho_{0}=1.1 \mathrm{Mg} / \mathrm{m}^{3}$, Poisson's ratio $\nu=0.499$ and $\alpha_{C F L}=0.3$. Stabilising parameters: $\tau_{\boldsymbol{F} p}=\Delta t, \tau_{\boldsymbol{p} J}=0.2 \Delta t, \alpha=0$ and $\beta=0.5$. Lumped mass contribution. 


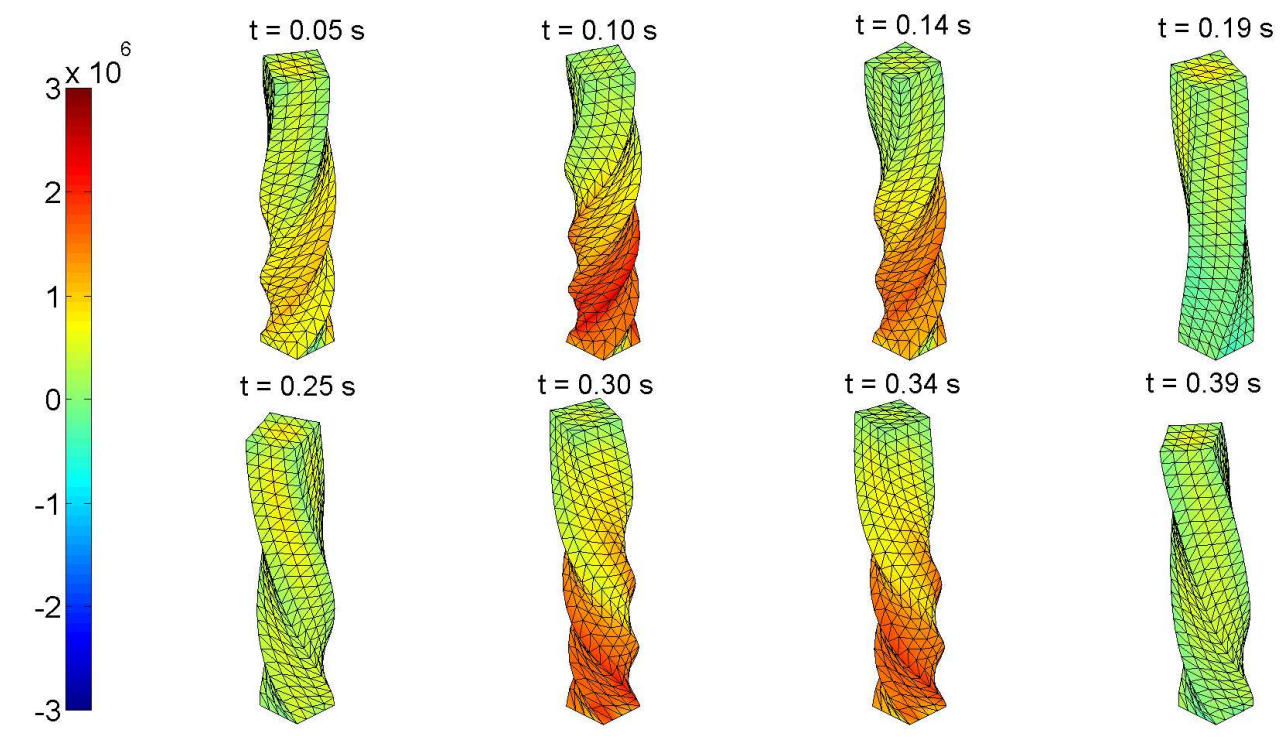

Figure 10: Twisting Column: Sequence of deformed shapes (pressure contour plot) using fractional step PG formulation. Results obtained with a initial sinusoidal rotational velocity $\Omega=100 \mathrm{rad} / \mathrm{s}$. A nearly incompressible NeoHookean $(\mathrm{NH})$ constitutive model is used such that Young's modulus $E=$ $0.017 \mathrm{GPa}$, density $\rho_{0}=1.1 \mathrm{Mg} / \mathrm{m}^{3}$, Poisson's ratio $\nu=0.5$ and $\alpha_{C F L}=0.3$. Stabilising parameters: $\tau_{\boldsymbol{F} \boldsymbol{p}}=\Delta t, \tau_{\boldsymbol{p} J}=0.2 \Delta t, \alpha=0$ and $\beta=0.5$. Lumped mass contribution.

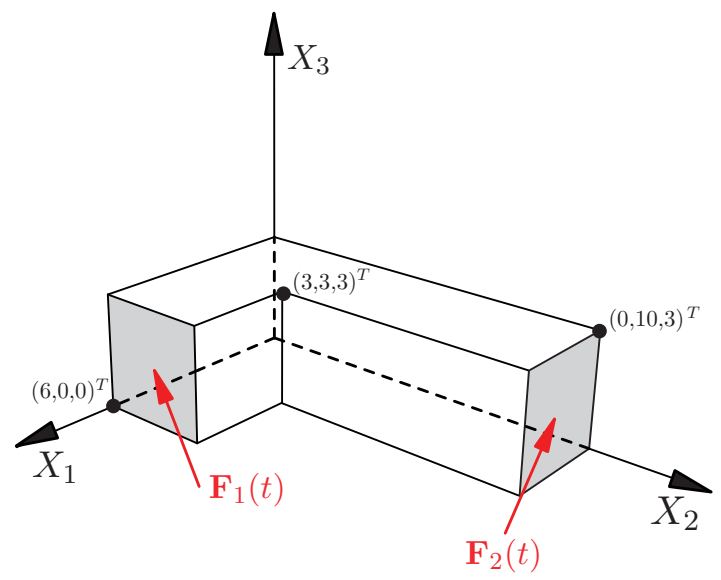

Figure 11: L-shaped block configuration 


\subsection{L-shaped block}

This benchmark problem, originally proposed by [78] and subsequently presented in [71, 78-80], is included in order to assess the ability of the algorithm to preserve angular momentum. We consider the motion of a threedimensional L-shaped block subjected to initial impulse traction boundary conditions at two of its sides described as follows (see Figure 11)

$$
\boldsymbol{F}_{1}(t)=-\boldsymbol{F}_{2}(t)=\left\{\begin{array}{lr}
\eta_{0} \times t, & 0 \leq t<2.5 \\
\eta_{0} \times(5-t), & 2.5 \leq t<5 \\
0, & t \geq 5
\end{array}\right.
$$

where $\eta_{0}=[150,300,450]^{T}$. The material response is governed by the NeoHookean model where its physical properties are Young's modulus $E=$ $50046 \mathrm{~Pa}$, density $\rho_{0}=1 \mathrm{Mg} / \mathrm{m}^{3}$ and Poisson's ratio $\nu=0.3$. Figure 12 presents the momentum evolution of the system calculated with the fractional step method. A sequence of deformed states is illustrated in Figure $13 \mathrm{a}$, each of which agrees very well with the results obtained using $\boldsymbol{p}$ - $\boldsymbol{F}$ formulation proposed in [44] (see Figure 13b).

\subsection{Tensile cube}

The objective of this three dimensional tensile cube problem is to demonstrate the performance of the nonlinear fractional step PG formulation when a tetrahedral mesh is used in an incompressible regime. A unit block clamped at the bottom (traction-free conditions for the rest of the boundaries) is sub-

jected to a sinusoidal variation in initial velocity field $\boldsymbol{v}^{0}=\left[0,0, v_{0} \sin \left(\frac{\Pi X_{3}}{2 L}\right)\right]^{T}$ (where $v_{0}=500 \mathrm{~m} / \mathrm{s}$ ) is compatible with the boundary. An incompressible Neo-Hookean (NH) constitutive model is employed where the Young's modulus $E=21 \mathrm{GPa}$, density $\rho_{0}=7 \mathrm{Mg} / \mathrm{m}^{3}$ and Poisson's ratio $\nu=0.5$. Figure 14 compares the pressure contour (being this variable the most sensitive to any stabilisation technique adopted) with and without the $\beta$ stabilisation parameter appearing in the term $p^{\text {st }}$ (see equation (79a)). Observe that the fractional step PG formulation incorporating $\beta$-term eliminates the appearance of non-physical mechanisms similar to hourglassing in the solution (see Figure 15).

\subsection{Benchmarked Taylor Impact problem}

The classical benchmarking example demonstrates the impact of a cylindrical copper bar of initial radius $r_{0}=0.0032 \mathrm{~m}$ and length $L_{0}=0.0324 \mathrm{~m}$, 


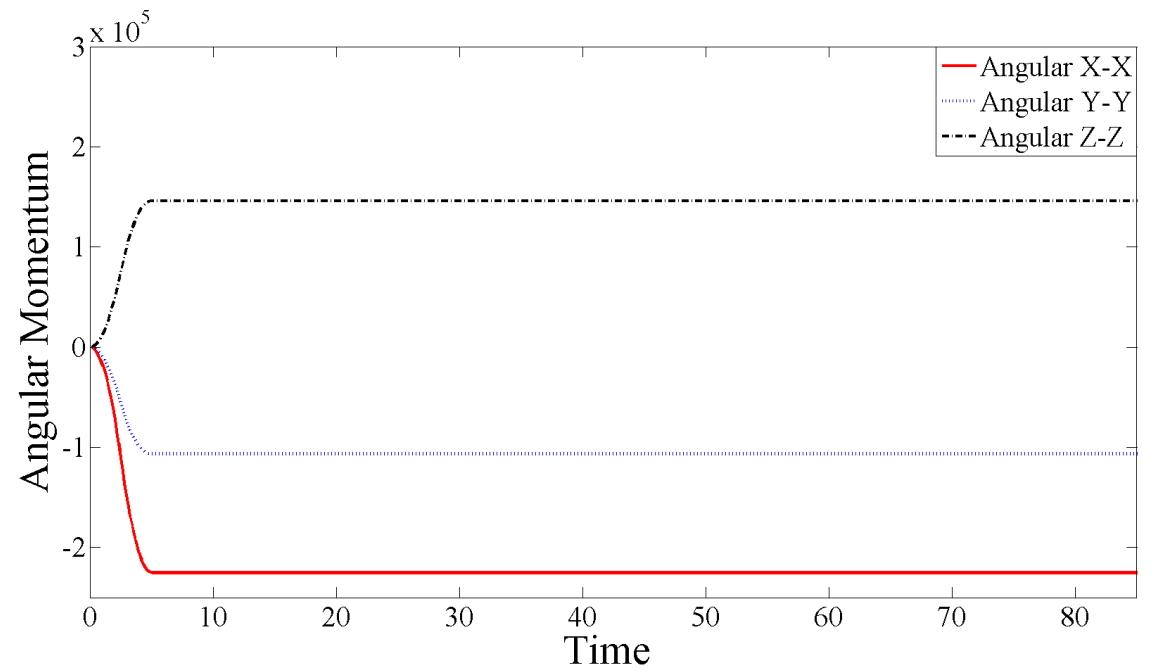

Figure 12: L-shaped Block: Results obtained with an impulse traction boundary conditions (86) at two of its sides using fractional step PG formulation. This example is run with the Neo-Hookean (NH) constitutive model and material properties are such that Young's modulus $E=50046 \mathrm{~Pa}$, density $\rho_{0}=1 \mathrm{Mg} / \mathrm{m}^{3}$, Poisson's ratio $\nu=0.3$ and $\alpha_{C F L}=0.3$. Stabilising parameters: $\tau_{\boldsymbol{F} \boldsymbol{p}}=\Delta t, \tau_{\boldsymbol{p} J}=0.2 \Delta t, \alpha=0$ and $\beta=0.5$. Lumped mass contribution. 


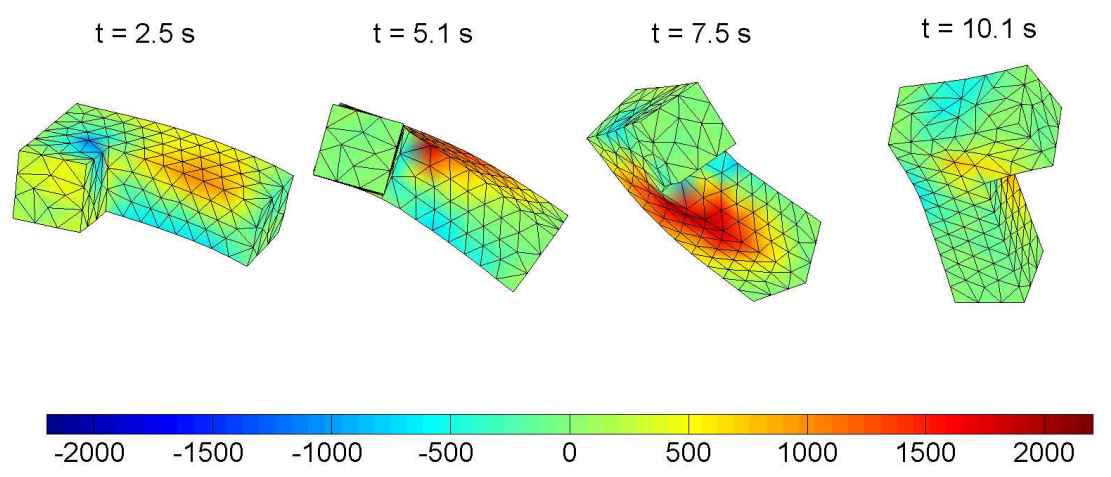

(a) Nonlinear fractional step PG formulation $\left(\tau_{\boldsymbol{F} \boldsymbol{p}}=\Delta t, \tau_{\boldsymbol{p} J}=0.2 \Delta t, \alpha=0\right.$ and $\left.\beta=0.5\right)$

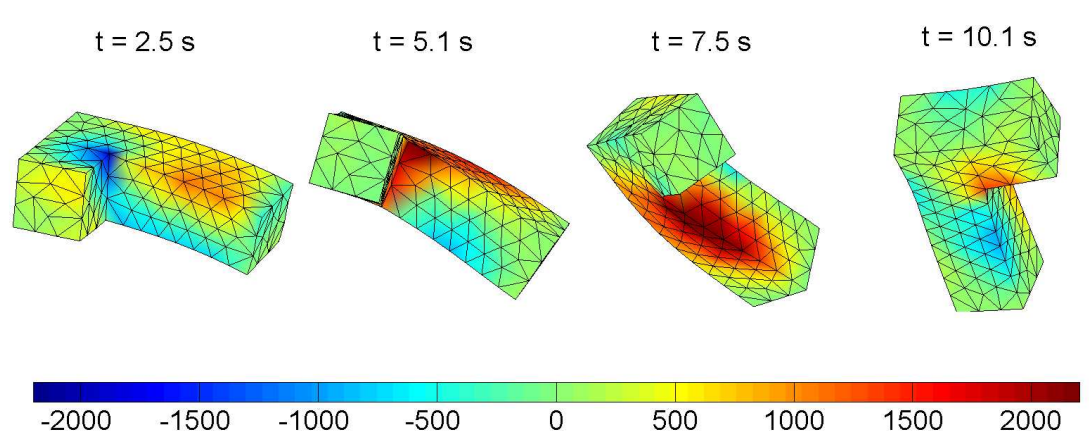

(b) Standard $\boldsymbol{p}-\boldsymbol{F}$ PG formulation $\left(\tau_{\boldsymbol{F}}=\Delta t, \tau_{\boldsymbol{p}}=0, \alpha=0.1\right)$

Figure 13: L-shaped Block: Results obtained with an impulse traction boundary conditions (86) at two of its sides using (a) Nonlinear fractional step PG formulation; and (b) Standard $\boldsymbol{v}-\boldsymbol{F}$ PG formulation. This example is run with the Neo-Hookean $(\mathrm{NH})$ constitutive model and material properties are such that Young's modulus $E=50046 \mathrm{~Pa}$, density $\rho_{0}=1 \mathrm{Mg} / \mathrm{m}^{3}$, Poisson's ratio $\nu=0.3$ and $\alpha_{C F L}=0.3$. Lumped mass contribution. 


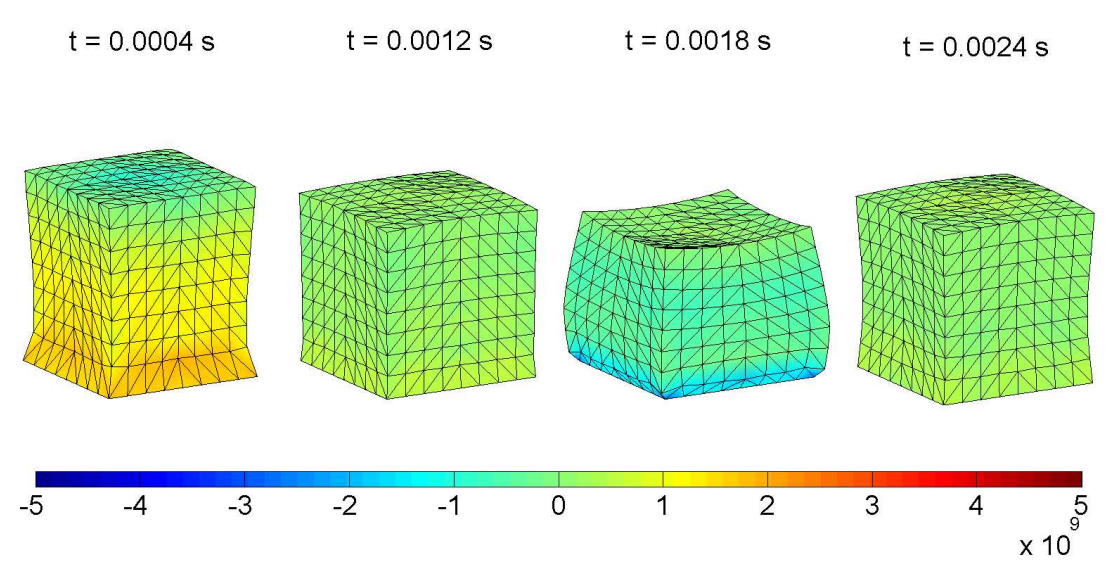

(a) Imposing stabilisation $\beta=0.5$

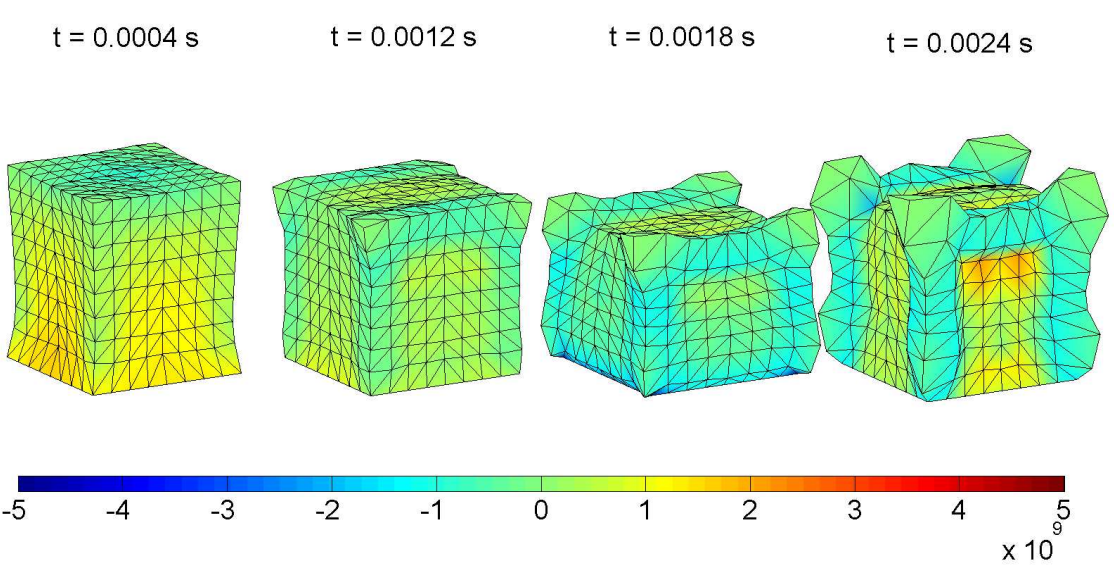

(b) Imposing stabilisation $\beta=0$

Figure 14: Tensile Problem: A sequence of deformed shapes (pressure contour plot) using nonlinear fractional step PG formulation: (a) With $\beta$ stabilisation; and (b) Without $\beta$ stabilisation. Results obtained with a sinusoidal variation in initial velocity field $\boldsymbol{v}^{0}=\left[0,0, v_{0} \sin \left(\frac{\Pi X_{3}}{2 L}\right)\right]^{T}$ where $v_{0}=500 \mathrm{~m} / \mathrm{s}$. Incompressible Neo-Hookean $(\mathrm{NH})$ constitutive model is used such that Young's modulus $E=21 \mathrm{~Pa}$, density $\rho_{0}=7 \mathrm{Mg} / \mathrm{m}^{3}$, Poisson's ratio $\nu=0.5$ and $\alpha_{C F L}=0.3$. Discretisation of $8 \times 8 \times 8 \times 6$ tetrahedral elements. Stabilising parameters: $\tau_{\boldsymbol{F} p}=\Delta t, \tau_{\boldsymbol{p}} \mathcal{2}=0.2 \Delta t$ and $\alpha=0$. Lumped mass contribution. 

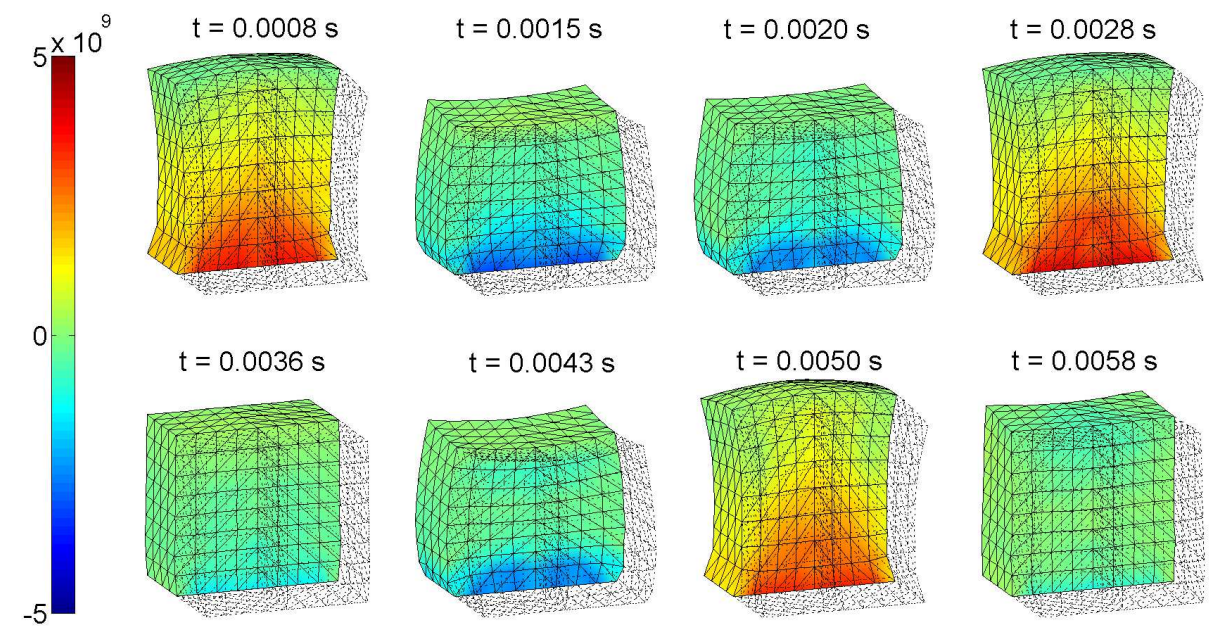

Figure 15: Tensile Problem: A sequence of cross-sectional deformed shapes (pressure contour plot) using nonlinear fractional step PG formulation. Results obtained with a sinusoidal variation in initial velocity field $\boldsymbol{v}^{0}=$ $\left[0,0, v_{0} \sin \left(\frac{\Pi X_{3}}{2 L}\right)\right]^{T}$ where $v_{0}=500 \mathrm{~m} / \mathrm{s}$. Incompressible Neo-Hookean $(\mathrm{NH})$ constitutive model is used such that Young's modulus $E=21 \mathrm{~Pa}$, density $\rho_{0}=7 \mathrm{Mg} / \mathrm{m}^{3}$, Poisson's ratio $\nu=0.5$ and $\alpha_{C F L}=0.3$. Discretisation of $8 \times 8 \times 8 \times 6$ tetrahedral elements. Stabilising parameters: $\tau_{\boldsymbol{F} \boldsymbol{p}}=\Delta t$, $\tau_{p J}=0.1 \Delta t, \alpha=0$ and $\beta=0.5$. Lumped mass contribution. 


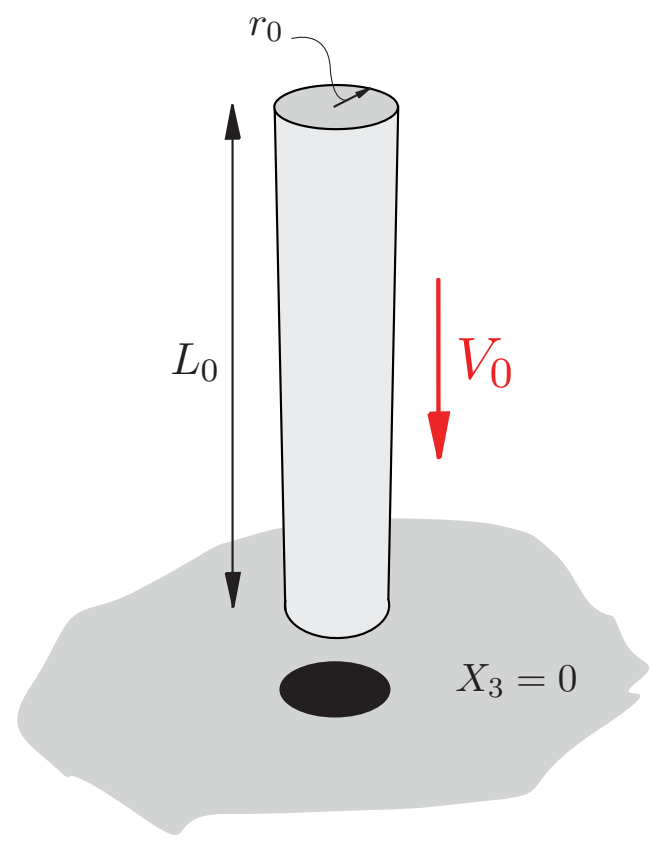

Figure 16: Classical benchmark impact bar configuration

against a rigid wall (see Figure 16). The bar is made of a nearly incompressible Neo-Hookean (NH) constitutive model such that Young's modulus $E=117 \mathrm{GPa}$ and density $\rho_{0}=8930 \mathrm{~kg} / \mathrm{m}^{3}$ and is dropped with a constant velocity $V_{0}=1000 \mathrm{~m} / \mathrm{s}$. The primary interest of this problem is to show the effectiveness of the fractional step method in both compressible and truly incompressible regimes, where in the latter case no volumetric deformation is allowed. Locking-free deformed sequences based on two different Poisson's ratios are presented in Figure 17. Observe that the pressure resolution is clearly freed from non-physical pressure checkerboard modes.

\section{Conclusions}

In this paper, a new computational methodology has been presented for the analysis of bending dominated nearly and truly incompressible large deformations in fast solid dynamics. The methodology is based upon a system of first order conservation laws, where the linear momentum $\boldsymbol{p}$ conservation equation is supplemented with two geometric conservation laws, one for the deformation gradient $\boldsymbol{F}$ and one for the Jacobian of the deformation $J$. A 


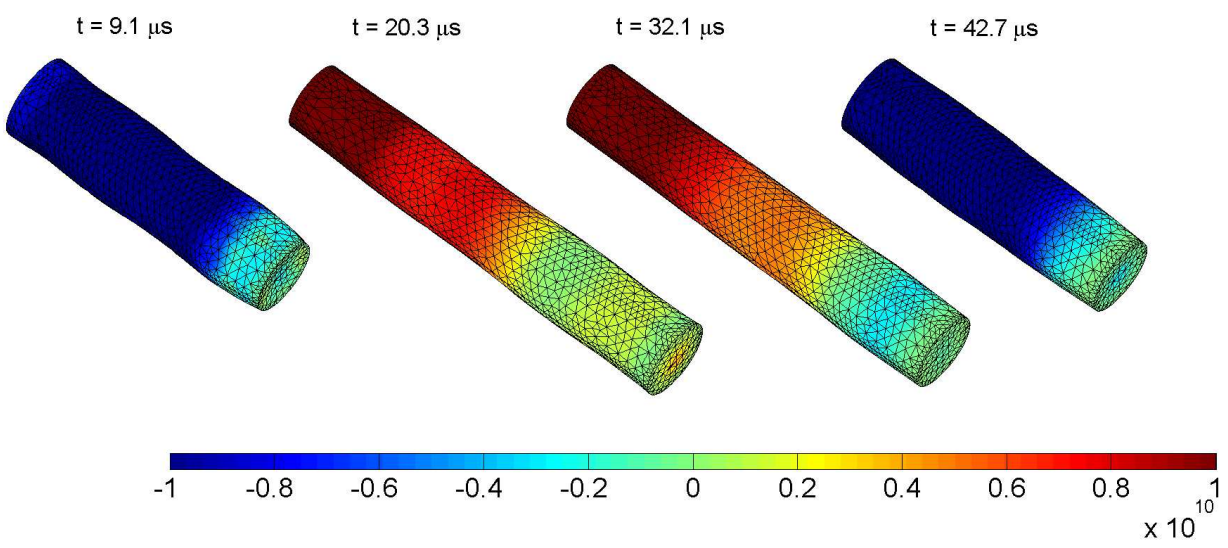

(a) Compressible regime (bulk modulus $\kappa=97.5 \mathrm{GPa}$ and shear modulus $\mu=45 \mathrm{GPa}$ )

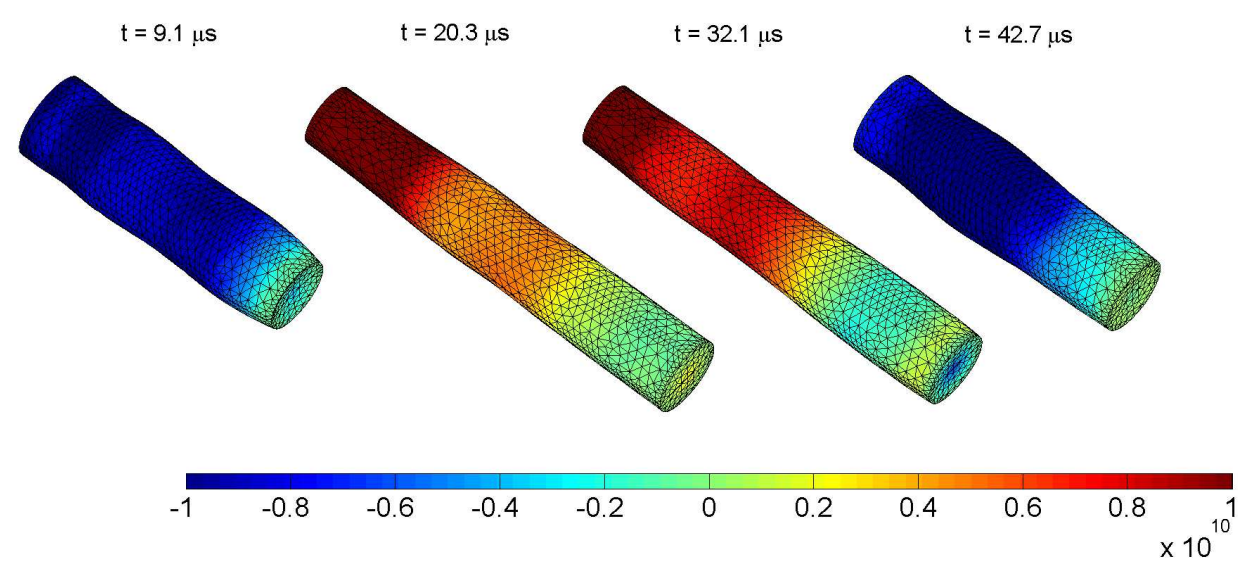

(b) Incompressible regime (bulk modulus $\kappa=\infty$ and shear modulus $\mu=39 \mathrm{GPa}$ )

Figure 17: Classical Taylor Impact Problem: Sequence of deformed shapes (pressure contour plot) using nonlinear fractional step PG formulation $\left(\tau_{\boldsymbol{F p}}=\right.$ $\Delta t, \tau_{\boldsymbol{p} J}=0.2 \Delta t, \alpha=0$ and $\beta=0.5$ ) for (a) Poisson's ratio $\nu=0.3$; and (b) Poisson's ratio $\nu=0.5$. Results obtained with a constant initial velocity $V_{0}=$ $1000 \mathrm{~m} / \mathrm{s}$. A nearly incompressible Neo-Hookean $(\mathrm{NH})$ constitutive model is used such that Young's modulus $E=117 \mathrm{GPa}$, density $\rho_{0}=8930 \mathrm{~kg} / \mathrm{m}^{3}$ and $\alpha_{C F L}=0.3$. Lumped mass contribution. 
Petrov-Galerkin spatial discretisation method has been employed for stabilisation of the governing equations and an adapted fractional step method has been presented when dealing with nonlinear deformations in the incompressible limit. Both velocities (or displacements) and stresses display the same rate of convergence, which proves ideal in the case of low order finite elements (usually preferred in commercial codes). It has been shown that the enhanced $\boldsymbol{p}$ - $\boldsymbol{F}$ - $J$ formulation overcomes locking and non-physical hydrostatic fluctuations in the pressure, providing a good balance between accuracy and speed of computation. The incorporation of polyconvex energy functionals $[81,82]$ into the existing mixed methodology is the next step of our work.

\section{Acknowledgements}

The first author acknowledges the financial support received through "The Leverhulme Prize" awarded by The Leverhulme Trust, United Kingdom.

\section{References}

[1] J. O. Hallquist, LS-DYNA theoretical manual, Livermore Software Technology Corporation (1998).

[2] J. O. Hallquist, LS-DYNA keyword user's manual, Livermore Software Technology Corporation (2003).

[3] D. J. Benson, Computational methods in Lagrangian and Eulerian hydrocodes, Computer Methods in Applied Mechanics and Engineering 99 (1992) 235-394.

[4] D. P. Flanagan, T. Belytschko, A uniform strain hexahedron and quadrilateral with orthogonal hourglass control, International Journal for $\mathrm{Nu}$ merical Methods in Engineering 17 (1981) 679-706.

[5] G. L. Goudreau, J. O. Hallquist, Recent developments in large scale Lagrangian hydrocodes, Computer Methods in Applied Mechanics and Engineering 33 (1982) 725-757.

[6] Hibbitt, Karlsson, Sorensen, ABAQUS/Explicit: User's manual (volume 1, version 6.1) (2000). 
[7] PAM-CRASH/PAM-SAFE: Solver reference with manual, PAM SYSTEM International (2002).

[8] D. J. Payen, K. J. Bathe, A stress improvement procedure, Computers and Structures 112-113 (2012) 311-326.

[9] D. J. Payen, K. J. Bathe, Improved stresses for the 4-node tetrahedral element, Computers and Structures 89 (2011) 1265-1273.

[10] C. H. Lee, A. J. Gil, J. Bonet, Development of a cell centred upwind finite volume algorithm for a new conservation law formulation in structural dynamics, Computers and Structures 118 (2013) 13-38.

[11] C. R. Dohrmann, M. W. Heinstein, J. Jung, S. W. Key, W. R. Witkowski, Node-based uniform strain elements for three-node triangular and four-node tetrahedral meshes, International Journal for Numerical Methods in Engineering 47 (2000) 1549-1568.

[12] T. Belytschko, W. K. Liu, B. Moran, Nonlinear finite elements for continua and structures, John Wiley and Sons, 2000.

[13] K. J. Bathe, Finite element procedures, Prentice Hall, 1996.

[14] N. M. Newmark, A method of computation for structural dynamics, Journal of the Engineering Mechanics Division 85 (1959) 67-94.

[15] T. J. R. Hughes, The finite element method: Linear static and dynamic finite element analysis, Dover Publications, 2000.

[16] H. M. Hilber, T. J. R. Hughes, R. L. Taylor, Improved numerical dissipation for time integration algorithms in structural dynamics, Earthquake Engineering and Structural Dynamics 5 (1977) 283-292.

[17] W. L. Wood, M. Bossak, O. C. Zienkiewicz, An alpha modification of Newmark's method, International Journal for Numerical Methods in Engineering 15 (1980) 1562-1566.

[18] J. Chung, G. M. Hulbert, A time integration algorithm for structural dynamics with improved numerical dissipation: The generalized $\alpha$ method, Journal of Applied Mechanics 60 (1993) 371-375. 
[19] D. D. Adams, W. L. Wood, Comparison of Hilber-Hughes-Taylor and Bossak $\alpha$ method for the numerical integration of vibration equations, International Journal for Numerical Methods in Engineering 19 (1983) 765-771.

[20] K. Washizu, Variational methods in elasticity and plasticity, Pergamon Press, Oxford, 1975.

[21] J. C. Nagtegaal, D. M. Park, J. R. Rice, On numerically accurate finite element solutions in the fully plastic range, Computer Methods in Applied Mechanics and Engineering 4 (1974) 153-177.

[22] J. H. Argyris, P. C. Dunne, T. Angelopoulos, B. Bichat, Large natural strains and some special difficulties due to nonlinearity and incompressibility in finite elements, Computer Methods in Applied Mechanics and Engineering 4 (1974) 219-278.

[23] D. S. Malkus, T. J. R. Hughes, Mixed finite element methods - Reduced and selective integration techniques: A unification of concepts, Computer Methods in Applied Mechanics and Engineering 15 (1978) 63-81.

[24] T. J. R. Hughes, Generalization of selective integration procedures to anisotropic and nonlinear media, International Journal for Numerical Methods in Engineering 15 (1980) 1413-1418.

[25] J. C. Simo, R. L. Taylor, K. S. Pister, Variational and projection methods for the volume constraint in finite deformation elasto-plasticity, Computer Methods in Applied Mechanics and Engineering 51 (1985) $177-208$.

[26] E. A. de Souza Neto, D. Peric, M. Dutko, D. R. J. Owen, Design of simple low order finite elements for large strain analysis of nearly incompressible solids, International Journal of Solids and Structures 33 (1996) 3277-3296.

[27] T. Sussman, K. J. Bathe, A finite element formulation for nonlinear incompressible elastic and inelastic analysis, Computers and Structures 26 (1987) 357-409. 
[28] B. Moran, M. Ortiz, C. F. Shih, Formulation of implicit finite element methods for multiplicative finite deformation plasticity, International Journal for Numerical Methods in Engineering 29 (1990) 483-514.

[29] J. C. Simo, F. Armero, Geometrically non-linear enhanced strain mixed methods and the method of incompatible modes, International Journal for Numerical Methods in Engineering 33 (1992) 1413-1449.

[30] J. Bonet, A. J. Burton, A simple average nodal pressure tetrahedral element for incompressible and nearly incompressible dynamic explicit applications, Communications in Numerical Methods in Engineering 14 (1998) 437-449.

[31] J. Bonet, H. Marriott, O. Hassan, An averaged nodal deformation gradient linear tetrahedral element for large strain explicit dynamic applications, Communications in Numerical Methods in Engineering 17 (2001) $551-561$.

[32] M. W. Gee, C. R. Dohrmann, S. W. Key, W. A. Wall, A uniform nodal strain tetrahedron with isochoric stabilization, International Journal for Numerical Methods in Engineering 78 (2009) 429-443.

[33] M. A. Puso, J. Solberg, A stabilized nodally integrated tetrahedral, International Journal for Numerical Methods in Engineering 67 (2006) 841-867.

[34] O. C. Zienkiewicz, J. Rojek, R. L. Taylor, M. Pastor, Triangles and tetrahedra in explicit dynamic codes for solids, International Journal for Numerical Methods in Engineering 43 (1998) 565-583.

[35] J. Bonet, H. Marriott, O. Hassan, Stability and comparison of different linear tetrahedral formulations for nearly incompressible explicit dynamic applications, International Journal for Numerical Methods in Engineering 50 (2001) 119-133.

[36] F. M. A. Pires, E. A. de Souza Neto, J. L. de la Cuesta Padilla, An assessment of the average nodal volume formulation for the analysis of nearly incompressible solids under finite strains, Communications in Numerical Methods in Engineering 20 (2004) 569-583. 
[37] T. J. R. Hughes, L. P. Franca, M. Balestra, A new finite element formulation for computational fluid dynamics: V. Circumventing the Babuška-Brezzi condition: a stable Petrov-Galerkin formulation of the Stokes problem accommodating equal-order interpolations, Computer Methods in Applied Mechanics and Engineering 59 (1986) 85-99.

[38] F. Brezzi, On the existence, uniqueness and approximation of saddlepoint problems arising from lagrangian multipliers, ESAIM: Mathematical Modelling and Numerical Analysis - Modélisation Mathématique et Analyse Numérique 8 (1974) 129-151.

[39] B. A. Szabo, I. Babuska, Finite element analysis, John Wiley and Sons, 1991.

[40] W. Elmer, J. S. Chen, M. Puso, E. Taciroglu, A stable, meshfree, nodal integration method for nearly incompressible solids, Finite Elements in Analysis and Design 51 (2012) 81-85.

[41] M. Cervera, M. Chiumenti, R. Codina, Mixed stabilized finite element methods in nonlinear solid mechanics: Part I: Formulation, Computer Methods in Applied Mechanics and Engineering 199 (2010) 2559-2570.

[42] F. Auricchio, L. B. da Veiga, C. Lovadina, A. Reali, R. L. Taylor, P. Wriggers, Approximation of incompressible large deformation elastic problems: Some unresolved issues, Computational Mechanics (2013) $1-15$.

[43] J. Schröder, P. Wriggers, D. Balzani, A new mixed finite element based on different approximations of the minors of deformation tensors, Computer Methods in Applied Mechanics and Engineering 200 (2011) 35833600 .

[44] C. H. Lee, A. J. Gil, J. Bonet, Development of a stabilised PetrovGalerkin formulation for conservation laws in Lagrangian fast solid dynamics, Computer Methods in Applied Mechanics and Engineering 268 (2014) 40-64.

[45] I. A. Karim, C. H. Lee, A. J. Gil, J. Bonet, A two-step Taylor Galerkin formulation for fast dynamics, Engineering Computations (2013). In press, DOI: 10.1108/EC-12-2012-0319. 
[46] G. Scovazzi, Lagrangian shock hydrodynamics on tetrahedral meshes: A stable and accurate variational multiscale approach, Journal of Computational Physics 231 (2012) 8029-8069.

[47] G. Scovazzi, M. A. Christon, T. J. R. Hughes, J. N. Shadid, Stabilized shock hydrodynamics: I. A Lagrangian method, Computer Methods in Applied Mechanics and Engineering 196 (2007) 923-966.

[48] G. Scovazzi, Stabilized shock hydrodynamics: II. Design and physical interpretation of the SUPG operator for Lagrangian computations, Computer Methods in Applied Mechanics and Engineering 196 (2007) 967-978.

[49] G. Scovazzi, J. N. Shadid, E. Love, W. J. Rider, A conservative nodal variational multiscale method for Lagrangian shock hydrodynamics, Computer Methods in Applied Mechanics and Engineering 199 (2010) 3059-3100.

[50] A. J. Gil, J. Bonet, J. Silla, O. Hassan, A Discrete Geometric Conservation Law (DGCL) for a cell vertex finite-volume algorithm on moving domains, International Journal for Numerical Methods in Biomedical Engineering 26 (2010) $770-779$.

[51] R. Courant, K. Friedrichs, H. Lewy, On the partial difference equations of mathematical physics, Mathematische Annalem 100 (1928) 32-74.

[52] A. J. Chorin, The numerical solution of the Navier-Stokes equations for an incompressible fluid, Bulletin of the American Mathematical Society 73 (1967) 928-931.

[53] A. J. Chorin, Numerical solution of the Navier-Stokes equations, Mathematics of Computation 22 (1968) 745-762.

[54] A. J. Chorin, On the convergence of discrete aapproximations to the Navier-Stokes equations, Mathematics of Computation 23 (1969) 341353.

[55] A. J. Gil, A. A. Carreño, J. Bonet, O. Hassan, The Immersed Structural Potential Method for haemodynamic applications, Journal of Computational Physics 229 (2011) 8613-8641. 
[56] A. J. Gil, , A. A. Carreño, J. Bonet, O. Hassan, An enhanced Immersed Structural Potential Method for fluid-structure interaction, Journal of Computational Physics 250 (2013) 178-205.

[57] S. K. Lahiri, J. Bonet, J. Peraire, L. Casals, A variationally consistent fractional time-step integration method for incompressible and nearly incompressible Lagrangian dynamics, International Journal for Numerical Methods in Engineering 63 (2005) 1371-1395.

[58] J. E. Marsden, T. J. R. Hughes, Mathematical foundations of elasticity, Dover Publications, 1994.

[59] T. J. R. Hughes, L. P. Franca, M. Mallet, A new finite element formulation for computational fluid dynamics: I. Symmetric forms of the compressible Euler and Navier-Stokes equations and the second law of thermodynamics, Computer Methods in Applied Mechanics and Engineering 54 (1986) 223-234.

[60] Q. Tiehu, Symmetrizing nonlinear elastodynamic system, Journal of Elasticity 50 (1998) 245-252.

[61] J. Bonet, R. D. Wood, Nonlinear continuum mechanics for finite element analysis, Cambridge University Press, second edition, 2008.

[62] T. J. R. Hughes, G. Scovazzi, T. E. Tezduyar, Stabilized methods for compressible flows, Journal of Scientific Computing 43 (2010) 343-368.

[63] T. J. R. Hughes, T. E. Tezduyar, Finite element methods for first-order hyperbolic systems with particular emphasis on the compressible Euler equations, Computer Methods in Applied Mechanics and Engineering 45 (1984) 217-284.

[64] T. J. R. Hughes, L. P. Franca, M. Mallet, A new finite element formulation for computational fluid dynamics: Vi. Convergence analysis of the generalized SUPG formulation for linear time-dependent multidimensional advective-diffusive systems, Computer Methods in Applied Mechanics and Engineering 63 (1987) 97-112.

[65] T. E. Tezduyar, T. J. R. Hughes, Development of time-accurate finite element techniques for first-order hyperbolic systems with particular emphasis on the compressible Euler equations, NASA Technical Report NASA-CR-204772, NASA (1982). 
[66] T. E. Tezduyar, T. J. R. Hughes, Finite element formulations for convection dominated flows with particular emphasis on the compressible Euler equations, in: Proceedings of AIAA $21^{\text {st }}$ aerospace sciences meeting, AIAA paper 83-0125, Reno, Nevada (1983).

[67] T. J. R. Hughes, Multiscale phenomena: Green's functions, the Dirichlet-to-Neumann formulation, subgrid scale models, bubbles and the origins of stabilized methods, Computer Methods in Applied Mechanics and Engineering 127 (1995) 387-401.

[68] T. J. R. Hughes, G. R. Feijóo, L. Mazzei, J.-B. Quincy, The variational multiscale method - a paradigm for computational mechanics, Computer Methods in Applied Mechanics and Engineering 166 (1998) 3-24.

[69] T. J. R. Hughes, G. Scovazzi, L. P. Franca, in: E. Stein, R. de Borst, T. J. R. Hughes (Eds.), Encyclopedia of Computational Mechanics, John Wiley and Sons, 2004.

[70] G. Scovazzi, E. Love, M. J. Shashkov, A multi-scale Q1/P0 approach to Lagrangian shock hydrodynamics, Computer Methods in Applied Mechanics and Engineering 197 (2008) 1056-1079.

[71] M. Aguirre, A. J. Gil, J. Bonet, A. A. Carreño, A vertex centred finite volume Jameson-Schmidt-Turkel (JST) algorithm for a mixed conservation formulation in solid dynamics, Journal of Computational Physics (2013). In press, DOI: 10.1016/j.jcp.2013.12.012.

[72] C. Shu, Osher, Efficient implementation of essentially non-oscillatory shock capturing schemes, Journal of Computational Physics 77 (1988) 439-471.

[73] R. Codina, Pressure stability in fractional step finite element methods for incompressible flows, Journal of Computational Physics 170 (2001) $112-140$.

[74] J. Donea, A. Huerta, Finite element methods for flow problems, Wiley and Sons, 2004.

[75] O. Gonzalez, A. M. Stuart, A first course in continuum mechanics, Cambridge University Press, 2008. 
[76] E. D. S. Neto, D. Perić, D. R. J. Owen, Computational methods for plasticity: Theory and applications, John Wiley and Sons, 2008.

[77] A. F. Bower, Applied mechanics of solids, CRC Press, 2010.

[78] J. C. Simo, N. Tarnow, A new energy and momentum conserving algorithm for the non-linear dynamics of shells, International Journal for Numerical Methods in Engineering 37 (1994) 2527-2549.

[79] L. Noels, L. Stainier, J. P. Ponthot, An energy-momentum conserving algorithm for non-linear hypoelastic constitutive models, International Journal for Numerical Methods in Engineering 59 (2004) 84-114.

[80] X. N. Meng, T. A. Laursen, Energy consistent algorithms for dynamic finite deformation plasticity, Computer Methods in Applied Mechanics and Engineering 191 (2002) 1639-1675.

[81] J. M. Ball, Convexity conditions and existence theorems in nonlinear elasticity, Archive for Rational Mechanics and Analysis 63 (1976) 337403.

[82] D. H. Wagner, Symmetric-hyperbolic equations of motion for a hyperelastic material, Journal of Hyperbolic Differential Equations 06 (2009) 615-630. 